This item was submitted to Loughborough's Research Repository by the author.

Items in Figshare are protected by copyright, with all rights reserved, unless otherwise indicated.

\title{
A study of computational methods for wake structure and base pressure prediction of a generic SUV model with fixed and rotating wheels
}

PLEASE CITE THE PUBLISHED VERSION

http://dx.doi.org/10.1177/0954407016685496

\section{PUBLISHER}

SAGE (@ the authors)

VERSION

AM (Accepted Manuscript)

\section{PUBLISHER STATEMENT}

This work is made available according to the conditions of the Creative Commons Attribution-NonCommercial 4.0 International (CC BY-NC 4.0) licence. Full details of this licence are available at: http://creativecommons.org/licenses/by-nc/4.0/

\section{LICENCE}

CC BY-NC 4.0

\section{REPOSITORY RECORD}

Forbes, David C., Gary J. Page, Martin A. Passmore, and Adrian P. Gaylard. 2016. "A Study of Computational Methods for Wake Structure and Base Pressure Prediction of a Generic SUV Model with Fixed and Rotating Wheels". figshare. https://hdl.handle.net/2134/23457. 


\title{
A study of computational methods for \\ wake structure and base pressure \\ prediction of a generic SUV model with \\ fixed and rotating wheels
}

\author{
David Forbes ${ }^{1}$, Gary Page ${ }^{1}$, Martin Passmore ${ }^{1}$ and Adrian Gaylard²
}

\begin{abstract}
This study is an evaluation of computational methods in reproducing experimental data for a generic SUV geometry and an assessment on the influence of fixed and rotating wheels for this geometry. Initially, comparisons are made in wake structure and base pressures between several CFD codes and experimental data. It was shown that steady-state RANS methods are unsuitable for this geometry due to a large scale unsteadiness in the wake caused by separation at the sharp trailing edge and rear wheel wake interactions. URANS offered no improvements in wake prediction despite a significant increase in computational cost. DES and Lattice Boltzmann methods showed the best agreement with experimental results in both wake structure and base pressure, with LBM running in approximately a fifth of the time for DES.
\end{abstract}

The study then continues by analysing the influence of rotating wheels and a moving ground plane over a fixed wheel and ground plane arrangement. The introduction of wheel rotation and a moving ground was shown to increase the base pressure and reduce the drag acting on the vehicle when compared to the fixed case. However, when compared to the experimental standoff case, variations in drag and lift coefficients were minimal but misleading, as significant variations to the surface pressures were present.

\section{Keywords}

Automotive, Vehicle Aerodynamics, Simulation, Unsteady, RANS, DES, Lattice-Boltzmann, SUV, Rotating Wheels, Moving Ground

\section{Introduction}

Over recent years, a rise in the popularity of sports utility vehicles (SUVs) has placed further importance on vehicle aerodynamics, as the large frontal area, high ground clearance and bluff profile of this vehicle type results in larger drag forces and increased emissions when compared to saloon and fastback geometries. This rise has been reflected in the recent work of Sterken et al. ${ }^{1-3}$ who have presented substantial experimental data for an in-production SUV with focus on understanding the wake structure, base pressure distribution and sources of drag. Wood et al. ${ }^{4}$ have also performed similar experimental work but for a generic, open-source SUV geometry whilst also investigating the effect of rear end geometry modifications in an attempt to lower the base drag contribution.
When compared to experimental testing, computational simulation has many benefits, with one of the largest being the wealth of information that a single simulation can provide. To capture the same amount of information in a wind tunnel requires the application of many techniques: Particle Image Velocimetry (PIV), surface pressures, hotwire and balance measurements. These methods can be time consuming to set up and also add to the underlying cost of the test. CFD simulation still has a setup time with preparation stages such as CAD modelling, spatial

\footnotetext{
${ }^{1}$ Loughborough University, UK

2 Jaguar Land Rover, UK
}

Corresponding author:

David Forbes, AAE Department, Stewart Miller Building, Loughborough University, Loughborough, UK.

Email: d.c.forbes@lboro.ac.uk 
discretisation and physics setup but can typically achieved in a much quicker time frame while the cost of simulation can depend on the code and methodology employed which can be inexpensive in comparison. The additional benefits can make computational simulation more favourable, however, it is important to validate what is fundamentally a prediction of the flow field and this data is provided by experimental testing. Therefore in the vehicle development process, it is common for both computational and experimental methods to be used in parallel.

One of the dangers when performing this type of validation, is for the analysis to focus solely on force coefficient values, predominantly the drag coefficient. Complementary drag coefficient values between experiment and simulation can imply an accurate prediction of the flow field, however in some cases, incorrect flow physics predictions in different regions of the vehicle can balance out, and fortuitously produce a misleadingly accurate coefficient value. Therefore when using CFD, is it crucial that a detailed analysis of flow is performed to ensure confidence in the prediction.

The main aim of this study is to present a computational comparison of the experimental work performed by Wood et al. ${ }^{4}$ using the generic SUV geometry, with specific focus on the accuracy of the wake structure and base pressure predictions. Initially, this study will compare CFD methodologies and their implementation within specific software packages to assess their suitability in predicting the experimental flow field. Although computational costs are included, the main purpose of this initial study is to present turbulence model comparison data for a simplified generic SUV model, rather than benchmarking the CFD codes. This study considers:

- Methodologies: Reynolds-Averaged Navier Stokes (RANS) and (URANS), Detached Eddy Simulation (DES) and the Lattice-Boltzmann Method (LBM).

- Software Codes: Commercial (CD-Adapco StarCCM+ v8.04.007), Open Source (OpenFOAM v2.1.1) and LBM Solver (Exa PowerFLOW v4.4d).

Comparison studies of this type have been performed in the past, however one which uses an open-access SUV geometry could not be found, making the proposed study unique. Nebenführ ${ }^{5}$ conducted a code comparison study using two codes and several full-scale vehicle models selected from the Volvo range. Whilst more recently Ashton and Revell ${ }^{6}$ and Ashton et al. ${ }^{7}$ conducted a thorough analysis of RANS and DES methods in predicting the flow around the Ahmed body and also, the realistic saloon style geometry of the DrivAer model. In their study, a large variety of RANS turbulence models and DES variants were assessed for their accuracy in predicting wake structure, base pressures and force coefficient values for both geometries. A similar study using the generic SUV geometry will complement this work well by bridging the large gap in geometry complexity from the Ahmed to the DrivAer bodies.

Following on from these results, a computational investigation into the effects of moving ground and rotating wheels will be performed. This type of simulation is becoming more relevant due to the introduction of the 'Worldwide Harmonised Light Vehicles Test Procedure' (WLTP) devised by the 'United Nations Economic Commission for Europe' (UNECE), which states that for determination of vehicle road load for use in emissions calculations, the aerodynamic drag obtained using wind tunnel facilities shall incorporate rotating wheels and a moving ground.

This condition is quite easily achieved in computational simulation through rotating wall boundary conditions, moving reference frames or overset grids and there are a wealth of studies which use such methods ${ }^{8-11}$, however the majority of studies use detailed, realistic geometries. The generic SUV with rotating wheels bridges the gap between low-fidelity, fundamental models such as the Ahmed and Windsor body ${ }^{12}$ and higher fidelity, realistic geometries. Hence these simulations should provide an insight into the more fundamental effects of wheel rotation without the interference of geometric features such as rim design and detailed underbody flows.

\section{Experimental Data}

The experimental data was obtained in the Loughborough University Model Scale (LU-MS) wind tunnel ${ }^{13}$, a lowturbulence tunnel with an average intensity of $0.15 \%$ in the core of the working section and tunnel area (TA) of $2.49 \mathrm{~m}^{2}$. The Generic SUV model, Figure 1, was designed within the Aeronautical and Automotive department at Loughborough University through analysis of the trends in SUV geometric features of the last forty years ${ }^{4}$. These trends were extrapolated to provide a prediction of a typical 2017 geometry. The model was designed to allow multiple configurations, however, the experimental aspect of this 
study concentrated on that with nominal ride height, smooth underbody, and fixed wheels. Table 1 provides dimensional data for the model.

[Insert Figure 1.]

[Insert Figure 2.]

Two dimensional, planar flow fields were recorded using PIV along six planes, (three vertical and two horizontal) in the vehicle's wake, which stretched downstream to a length of approximately $40 \%$ of the total vehicle length, Figure 1. Base and diffuser surface pressure values were recorded using an array of 75 pressure tappings, 63 on the base and 12 on the diffuser. Due to the scale of the model and size of the instrumentation located inside the model, tappings were limited to half of the models base and diffuser surfaces. Hence total surface data is subject to interpolation errors and assumes a symmetric base and diffuser pressure distribution. Finally, a six-component, underfloor, virtual centre balance measured aerodynamic force and moment data.

The model is $1 / 4$ scale with blockage of $5.4 \%$ in the LUMS tunnel test section and was run at a Reynolds number of $2.85 \times 10^{6}$ based on a reference length of $1.04 \mathrm{~m}$ (total model length). Surface pressures and forces were corrected for blockage effects using the MIRA correction based on continuity, Equations 1 and 2.

$$
\begin{gathered}
\mathrm{C}_{\mathrm{p}_{\text {cor }}}=1-\frac{1-\mathrm{C}_{\mathrm{p}}}{\left(1-\frac{\mathbf{A}}{\mathbf{T A}}\right)^{-2}} \\
\mathrm{C}_{\mathrm{D}_{\text {cor }}}=\frac{\mathrm{C}_{\mathrm{D}}}{\left(1-\frac{\mathbf{A}}{\mathbf{T A}}\right)^{-2}}
\end{gathered}
$$

A fixed ground plane in the tunnel required non-rotating wheels and development of a floor boundary layer. No boundary layer reduction devices were employed and previous measurements in an empty tunnel at model centre have recorded thicknesses $\left(\delta^{99}\right)$ of around $60 \mathrm{~mm}$. Coincidently, this height corresponds to the clearance height between the floor and SUV body, hence the flow in the underbody region will be strongly influenced by this tunnel boundary layer. Wheels were not in contact with the ground to ensure balance measurements were not influenced by any grounding of the model and all comprised of a flat on the tyre surface to simulate a typical contact patch. The clearance between the bottom of the tyre and ground was measured at $4 \mathrm{~mm}$, Figure 2.

\begin{tabular}{|c|c|}
\hline Length $(\mathbf{L})$ & $1.040 \mathrm{~m}$ \\
\hline Width & $0.410 \mathrm{~m}$ \\
\hline Height & $0.376 \mathrm{~m}$ \\
\hline Wheelbase & $0.650 \mathrm{~m}$ \\
\hline Ride Height & $0.060 \mathrm{~m}$ \\
\hline Wheel Diameter & $0.160 \mathrm{~m}$ \\
\hline Frontal Area $(\mathbf{A})$ & $0.139 \mathrm{~m}^{2}$ \\
\hline Diffuser Angle & $29.3^{\circ}$ \\
\hline Re $(\mathbf{L}, 40 \mathrm{~m} / \mathrm{s})$ & $2.85 \times 10^{6}$ \\
\hline
\end{tabular}

Table 1. Generic SUV Dimensions.

\section{CFD Procedure}

The dimensions of the CFD domain matched those of the wind tunnel working section to maintain the blockage of $5.4 \%$ present in the experiment. This resulted in a CAD model of approximately $3.6 \mathbf{L} \times 1.96 \mathbf{L} \times 1.32 \mathbf{L}$ in which the SUV geometry was positioned. The length of the working section model was then extended to $28 \mathbf{L}$, approximately $15.5 \mathbf{L}$ upstream and $12.5 \mathbf{L}$ downstream of the model centre, to avoid interference effects from the inlet and outlet boundary conditions. The domain is illustrated in Figure 3.

\section{[Insert Figure 3.]}

\section{Lattice Boltzmann Method}

The Lattice-Boltzmann Method (LBM) is a special discretisation of the Boltzmann equation in space, time and velocity. It simulates the flow and collision processes of particles within a Newtonian fluid ${ }^{14}$. The turbulence model employed in the code is similar to a Very-Large Eddy Simulation (VLES) with a k- $\epsilon$ RNG model acting as a sub-grid scale model. The LBM solves the transient, turbulent flow of air around the SUV and then the solution is averaged in time to give the mean flow solution.

Spatial discretisation in PowerFLOW generates a lattice, containing 'voxels' (cuboidal volume cells) and 'surfels' (surface cells generated as a voxel intersect a surface). The lattice used for this study was generated using industrial guidance, with automated voxel sizes (smallest element $1 \mathrm{~mm}, 0.001 \mathbf{L}$ ) and refinement regions based upon model dimensions. These 'best practices' apply to full-scale vehicles and large Reynolds numbers, however a quarter scale model a flow speed of $40 \mathrm{~m} / \mathrm{s}$ approximates to $32 \%$ full scale Reynolds number at typical motorway speeds. Hence the scaled down lattice should provide good resolution of small scale turbulence. A cross-section of the lattice shows the refinement around the vehicle and the sizes of the 
voxels within these regions (Figure 4a). The total number of voxels in the fluid domain is approximately 70 million with non-dimensional near wall spacings $\mathrm{y}^{+} \leq 50$ over the surfaces of the SUV and $\mathrm{y}^{+} \leq 700$ over the floor and tunnel walls. Thus wall functions are used to predict the boundary layers formed over these surfaces.

\section{Finite Volume Codes}

For RANS and URANS solvers, the k- $\epsilon$ realizable turbulence model with wall functions was chosen based on preliminary turbulence model comparisons alongside a second order upwind convection scheme. For DES, the IDDES formulation of the Spalart-Allmaras Detached Eddy Model was chosen, as this model is available in the specified builds of the two finite volume codes, with a hybrid second order upwind/bounded-central differencing convection scheme. These two FV codes employ a semi-implicit, pressure based, low-speed solver representative of the majority of commercial and academic codes. For both FV codes and each methodology, simulations were ran for the same number of iterations/timesteps whereas the stopping criterion for the LBM methodology was determined by monitoring the backward average of the force coefficients and best practices provided by industrial guidance.

A hexahedral dominant mesh was generated using the inbuilt trimmer mesher of the commercial code. This method, with similar refinement locations and isotropic cell sizes, allows the generation of a volume mesh with similar properties to the LBM lattice, Figure 4b. However differences were introduced at the wall boundaries. Fine wall resolution can be achieved using fewer cells by increasing their aspect ratio through prism layers, Figure 5, a property not available in the lattice approach due to the isotropic nature of voxels. The non-dimensional near wall spacing on the surfaces of the SUV was maintained at $\mathrm{y}^{+} \leq 50$, however the spacings on the tunnel walls were reduced $y^{+} \leq 100$. As a result wall functions are still used to predict the boundary layers formed over these surfaces. The total number of volume elements in the mesh is comparable to that of the lattice at 71 million. Once generated, the mesh was converted into a format that could be used by the open source code. Consequently, identical meshes could be run on the two FV codes for all methodologies, a desirable condition for careful comparison.

\section{[Insert Figure 5.]}

The second stage of this study concerns a moving ground plane and rotating wheels and as a result small modifications are made to the FV mesh. The vehicle is dropped to ensure contact between the base of the wheels and tunnel floor, Figure 6. However, this alteration does not cause a significant change in the mesh refinement or total number of elements.

\section{[Insert Figure 6.]}

\section{Boundary and Initial Conditions}

In order to correctly simulate the wind tunnel tests, the wind tunnel boundary layer has to be accurately captured. As the contraction section of the tunnel is not included in the CAD model, the point at which the boundary layer starts to develop can be calculated using flat plate boundary layer theory compared to an experimental thickness measurement in the working section. This appropriate length was found to be $4.1 \mathrm{~m}$ upstream of the model centre and thus, no-slip wall conditions are applied on all four surfaces accordingly. Slip wall conditions are then applied from this point forward to the velocity inlet, this region is outlined in Figure 3. Values for free stream velocity and turbulence intensity are set to $40 \mathrm{~m} / \mathrm{s}$ and $0.15 \%$ respectively and Reynolds number is maintained at $2.85 \times 10^{6}$ based on model length.

For the first stage of this study wheels were fixed and not in contact with the ground, however, for the second stage in which the effect of a moving ground and rotating wheels (MG\&RW) is investigated, wheels are in contact and moving ground and wheel rotation is imparted via tangential wall velocities. The velocity applied to the MVG matches the freestream velocity of $40 \mathrm{~m} / \mathrm{s}$ and wheel rotation rate was calculated based upon this ground velocity and the wheel radius. The rotating wall method was chosen for the wheels due to their geometric simplicity without wheel spokes. Alternative methods such as multiple reference frames (MRF) and sliding mesh are available and for production type wheels on detailed vehicle geometry, differences have been shown in the accuracies of these methods $9 ; 10 ; 15$ but these were attributed to crossflow through the wheels and influence of axis asymmetric design. 


\section{Results}

\section{Code Comparison}

The test matrix of methodology and code configurations can be seen in the first two rows of Table 2, showing a total of seven simulations. Due to the size of this matrix and quantity of flow visualisation results, it is unfeasible to present all of the collected data in this paper and hence only the most important results feature. Data is presented in horizontal and vertical cut planes in the wake of the vehicle. The flow is moving from left to right, the base of the SUV is located at $X=0.52$ and all velocities are normalised by the freestream value of $40 \mathrm{~m} / \mathrm{s}$. The same plotting software is used to present both experimental and computational results.

\section{Steady State Solver}

The flow field taken from the commercial RANS methodology is shown in Figure 7. The wake flow is defined by two regions: a large recirculating region behind the base caused by flow separation at the sharp rear edges and a high velocity region as underbody flow is accelerated by the steep diffuser. Figure 8, shows a wake flow comparison between experimental results and codes employing steady state RANS governing equations. Two contours of normalized streamwise $u$ velocity are shown on a vertical plane, $Y=0$, through the centreline of the wake. The comparison suggests that the quantity of high velocity flow accelerated by the smooth underbody and diffuser is overpredicted by the RANS methodology. As a result, the size of the recirculating flow region behind the base is reduced when compared to the experiment. Further analysis of the flow in this region, Figure 9, reveals flow separation towards the top of the diffuser and thus is an area where the use of wall functions is not best suited as the first cell is no longer located in the log-law region of the boundary layer. No experimental data of the flow in this region has been taken but from the contouring in Figure 8 it is reasonable to suggest that the flow is attached and thus, is inaccurately predicted by a RANS methodology. Between the two codes, there is a slight variation in the direction and quantity of this diffuser flow which is most likely due to differences in the implementation of the walls functions in this region.

[Insert Figure 7.]

[Insert Figure 8.]

[Insert Figure 9.]

[Insert Figure 10.]

[Insert Figure 11.]
A second vertical plane outwards from the centreline, $Y=0.17$ Figure 10, directly in the wake of the right hand side wheels, demonstrates the deficiencies of the turbulence model in capturing what is potentially a highly unsteady region. The solutions have failed to capture accurately the shape of the time-averaged low velocity structure found in the experiment, with both codes over-predicting the size of the recirculating flow region. Despite these differences, a high similarity between the two computational flow fields exists, suggesting that the RANS methodology is the cause of the inaccuracies and not its implementation by the two codes.

A horizontal plane $Z=0.187$, immediately above the diffuser, highlights the weakness of RANS for this application further, Figure 11. The low velocity horseshoe structure found in the experiment, that is a result of the rear wheel wakes, is again overpredicted in both size and quantity of recirculating flow.

These results are comparable to the findings of Ashton et al. ${ }^{7}$ who showed that a RANS methodology over-predicted the size of the recirculation region in the wake of an Ahmed body. The authors discovered that this was due to an under-prediction of the turbulent kinetic energy in the initial separated shear layer on the $25^{\circ}$ top-roof slant. Although no turbulent kinetic energy comparisons are presented for this SUV study, the similarities in the rear end geometry, specifically the $30^{\circ}$ slant of the diffuser and vertical base surface, should make the conclusions transferable.

Although not included, periodic oscillations in the force coefficient histories, also point towards a large-scale unsteadiness in the flow that a RANS methodology is unable to capture. The natural progression is therefore to adopt an unsteady methodology that should capture these time-dependent fluctuations in the flow and hence show improvements over the current results.

\section{Unsteady Methodologies}

Time-averaged results from the URANS methodologies are shown in Figure 12. Figure 12a shows a contour comparison between RANS and URANS results in the vertical plane $Y=0.17$. The unsteady method displays minimal differences from the steady state results. This was not anticipated as it was expected that the unsteady solver would be able to capture a large scale unsteadiness caused by rear wheel wakes. Figure $12 \mathrm{~b}$ shows a comparison of $u$ 


\begin{tabular}{|l|c|c|c|c|c|c|c|c|}
\hline & \multirow{2}{*}{ Exp } & \multicolumn{3}{|c|}{ commercial } & \multicolumn{3}{c|}{ Open Source } & \multirow{2}{*}{ LBM } \\
\cline { 3 - 9 } & & RANS & URANS & DES & RANS & URANS & DES & \\
\hline $\mathrm{C}_{\mathrm{D}}$ & 0.445 & 0.403 & 0.400 & 0.453 & 0.413 & 0.417 & 0.454 & 0.434 \\
\hline Base $_{\mathrm{D}}$ & 0.203 & 0.173 & 0.171 & 0.194 & 0.168 & 0.170 & 0.196 & 0.194 \\
\hline $\mathrm{C}_{\mathrm{L}}$ & 0.041 & -0.042 & -0.037 & -0.166 & -0.057 & -0.052 & -0.132 & -0.087 \\
\hline
\end{tabular}

Table 2. Averaged Force Coefficients.

velocity along a horizontal line probe spanning the width of the wake. The line probe is located at a height coinciding with the horizontal plane $Z=0.187$ and an $X=0.63$ location coinciding with the location of minimum velocity recorded in the experiment. As consistent with the vertical contour plot, URANS offers very little difference from RANS and moves no closer towards the experimental flow field.

\section{[Insert Figure 12.]}

Alternative unsteady solvers are Detached Eddy Simulation (DES) and the Lattice Boltzmann Method (LBM). An instantaneous snapshot of the flow field taken from the commercial DES simulation is shown in Figure 13. A volume threshold where $-0.5 \leq c_{p_{\text {total }}} \leq 0$ is useful in identifying wake structures, this threshold is then rendered using values of $c_{p_{\text {total }}}$. It is clear that the DES methodology predicts a highly unsteady flow field and shows a strong interaction between the rear wheel wakes and base wake flow.

\section{[Insert Figure 13.]}

The time average of this flow field alongside open source DES and LBM results is shown in Figures 14, 15 and 16. The flow structures predicted by the DES and LBM approaches more accurately represent those found in the experiment in both size and shape of the dominating low velocity structure and offer considerable improvements over the steady state RANS results, especially in the $Y=0.17$ and $Z=0.187$ planes. Again, this is consistent with the work of Ashton et al. $^{7}$, who showed that switching to a DES methodology led to a more accurate prediction of the turbulent kinetic energy over the Ahmed body top-slant and consequently improvements in the wake velocity distribution.

Importantly, the Lattice-Boltzmann approach shows good agreement with these more established finite volume methods. As in the RANS cases, open source and the commercial solver results show small differences in the centreline diffuser flow, with commercial again overpredicting the size and incorrectly placing this high velocity region. It was suggested for the RANS cases that this may be due to differences in the implementation of the wall functions on the SUV surfaces, leading to variations in the underbody flow. This may still be the case as both unsteady codes are still employing RANS wall functions in these regions as part of the hybrid RANS/LES methodology.

\section{[Insert Figure 14.]}

[Insert Figure 15.]

[Insert Figure 16.]

Figures 17, 18 and 19 show comparisons of $u$ velocity along two vertical and one horizontal line probe. These probes are constrained to the PIV planes and their $X$ position coincides with minimum experimental velocity found in the respective PIV plane. It is clear that all simulations show good agreement with the experiment velocity distribution and differences between the predictions are mainly restricted to the flow behaviour towards the ground where the underbody and diffuser flows are most influential.

\section{[Insert Figure 17.]}

[Insert Figure 18.]

[Insert Figure 19.]

\section{Base Surface Pressures}

Figure 20 displays the time averaged pressure coefficient distribution on the SUV base surface. The experimental data was obtained using an array of 63 pressure tappings located on right hand side of the base and diffuser surface, thus the experimental pressure distribution is assumed symmetric. Due to their restricted numbers, interpolation errors will also exist when plotting the surface. Both experimental and computational data has been corrected for blockage effects using the MIRA method, Equation 1. All unsteady CFD methodologies are in good agreement with each other and the experiment in terms of pressure values and distribution. The effects of the wheels wakes are clearly identified in the contouring on the base surface. This again demonstrates the dependency of flow field on the accuracy of these flow 
structures. As expected due to the lack of accuracy in the wake flow prediction, the RANS methodology has failed to capture an acceptable distribution over the base surface and URANS offers very little improvement.

\section{[Insert Figure 20.]}

Table 2 shows lift and drag coefficients for the experiment and computations, all values corrected for blockage effects. The poor wake structure and base surface pressure prediction of the RANS results is reflected in the drag values. The two DES and LBM results are in much better agreement, with errors of approximately $2 \%$ to the total drag value and considerably less to the base contribution. Agreement with lift, however, is much less encouraging.

All configurations predict a negative lift coefficient value when the experiment shows a small positive lift. The predicted values also vary significantly between codes and methodologies. This is most likely due to inaccuracies in the underbody flow prediction over the smooth, flat surface of the SUV and a possible result of differences and inaccuracies in the implementation of the wall functions. Evidence of this has been seen in the diffuser flow, which differs between codes and methodologies to the experiment.

\section{Computational Resources}

The two finite volume codes were run on the HPC-Midlands facility, whilst the LBM code was run on an industrial facility. In all cases computations were run in parallel using 192 cores, although there were some differences in processor speeds. The HPC-Midlands processors were $2.0 \mathrm{GHz}$ Intel 'Sandy Bridge E5-2650' whilst the industrial facility used $2.93 \mathrm{GHz}$ Intel 'Xeon X5670' processors. Run times are presented in Tables 3 and 4, where the speed up parameter is normalised by the total run times of the commercial code RANS and DES respectively. Due to the differences in clock speed and total simulated time for the LBM case, the normalised speed up for the LBM has been scaled up by $\frac{2.93}{2.0} \times \frac{1}{0.279}=5.25$ to represent the value should the LBM code have been run on the same processors and for the same simulation time.

For the RANS method, the point of convergence is reached once the drag and lift force coefficient values have stabilized. This occurred at 5000 iterations for both the commercial and open source codes with similar run times. This result is reflected in the good similarities between the wake flow and base pressures previously discussed.

For the unsteady methods, this point corresponds to the initial transient and is obtained through backward averaging of the coefficients. Once this point has been reached, the simulation continues to run for a sufficient number of time steps to obtain a reliable averaged flow field. The averaging window for the LBM code was smaller than that of the finite volume codes and this has been accounted for in the normalised speed up value. It is appropriate to use the RANS solutions as an initialization for the unsteady methods and this has been done for all solvers using the finite volume codes. The time step for LBM is approximately an order of magnitude smaller than that used for DES which in turn, is an order of magnitude smaller than URANS. These have been calculated based on the scales of turbulence that are being resolved in the individual solvers. Finally, for the finite volume codes, five sub iterations were used per time step to encourage convergence within each time step.

In spite of the smaller time step value and hence greater number of time steps to an averaged solution for the LBM solver over DES, the former is computed in approximately a fifth of the time (accounting for differences in clock speed and simulated time). It may be expected that this is reflected by inaccuracies in the flow field, but as already shown wake structure and base pressure are highly comparable.

\section{An Assessment of Moving Ground and Rotating Wheels}

It has been found by the code and methodology comparison that for this geometry, the wheel wakes (and their entrainment by the high velocity diffuser flow into the base wake) have a strong influence on the overall wake structure and base pressure. With this in mind, it is reasonable to suggest that rotation of the wheels will have a large impact on the base pressure, due to the differences in wheel flow behaviour that rotation has shown in the past $^{8: 15-18}$.

For this reason, the study now progresses to assess the influence of moving ground and rotating wheels in detail. The analysis focuses on the effect of moving ground and rotating wheels (MG\&RW) in the base wake region when compared to a traditional fixed ground and fixed wheel (FG\&FW) arrangement. In both simulations, wheels were in contact with the floor and unlike the 


\begin{tabular}{|l|c|c|}
\hline & commercial RANS & Open Source RANS \\
\hline Elapsed time per iteration (secs) & 6.9 & 6.45 \\
\hline Number of Iterations to convergence & 5,000 & 5,000 \\
\hline Total time to convergence (secs) & 34,500 & 32,250 \\
\hline Normalised Speed Up & $\mathbf{1}$ & 1.07 \\
\hline
\end{tabular}

Table 3. RANS Computational Costs and Run Times.

\begin{tabular}{|l|c|c|c|c|c|}
\hline \multirow{2}{*}{} & \multicolumn{2}{|c|}{ commercial } & \multicolumn{2}{c|}{ Open Source } & \multirow{2}{*}{ LBM } \\
\cline { 2 - 5 } & URANS & DES & URANS & DES & \\
\hline Computational time step (s) & $2.5 \times 10^{-4}$ & $2.5 \times 10^{-5}$ & $2.5 \times 10^{-4}$ & $2.5 \times 10^{-5}$ & $2.23 \times 10^{-6}$ \\
\hline Elapsed time per $\Delta \mathrm{t}(\mathrm{s})$ & 15.35 & 17.82 & 14.2 & 14.84 & 0.22 \\
\hline Physical time averaging window (s) & 1 & 1 & 1 & 1 & 0.279 \\
\hline Number of time steps in averaging window & 4,000 & 40,000 & 4,000 & 40,000 & 125,112 \\
\hline Elapsed time for averaging window (s) & 61,400 & 712,800 & 56,800 & 593,600 & 27,525 \\
\hline Normalised Speed Up & 11.61 & $\mathbf{1}$ & 12.55 & 1.2 & 4.93 \\
\hline
\end{tabular}

Table 4. Transient Solvers Computational Costs and Run Times.

fixed floor, wheel standoff arrangement (WSO) simulated previously, experimental comparison data is not available for comparison.

Using the results from the CFD methodology study which showed the unsuitability of RANS and URANS methodologies for this geometry, all simulations were performed with the commercial code's unsteady DES solver. Boundary conditions and solver parameters have been described previously and all results have been time averaged over a simulated time of one second.

Initial comparisons on the base and diffuser surfaces suggest that the inclusion of wheel rotation provides a pressure recovery over the rear of the model. This is shown in Figure 21a by the positive $\Delta C_{p}=C_{p}^{\mathrm{MG} \& \mathrm{RW}}-C_{p}^{\mathrm{FG} \& \mathrm{FW}}$ over the base and surfaces, implying a higher pressure in the MG\&RW case. This is consistent with the well established experimental work of Elofsson and Bannister ${ }^{16}$, who displayed complementary positive pressure delta regions for a generic square back geometry with the introduction of rotating wheels.

\section{[Insert Figure 21.]}

The increase in pressure on the base surface leads to a reduction in base drag of 3 counts, presented in Table 5. However, this only contributes to approximately $20 \%$ of a global, 15 count, drag reduction, suggesting that the moving geometry has an influence on drag production elsewhere. On the diffuser surface, a reduction of five counts was calculated using projection from the thirty degree angled surface onto the drag axis. Thus together, the base and diffuser surfaces account for approximately half of the total drag reduction found by including MG\&RW.

The value of overall drag reduction (15 counts) is in good agreement with existing MG\&RW studies. Across a range of different geometries, drag reductions of up to 20 counts have been measured ${ }^{8 ; 17 ; 18}$. Thus a reduction of 15 counts for the generic SUV seems reasonable.

Figure 22a shows the differences in underbody pressure distribution, where the pressure difference within the wheel arches have been made visible by removing each wheel geometry from the images. The rotation of the wheels leads to a higher pressure region at the front and lower pressure region at the rear of each wheel arch when compared to the FG\&FW case. This effect is strongest in the front wheel arches as these wheels are exposed to highly accelerated flow as a result of the substantial taper angle of the body's front overhang which is typical of a SUV geometry. Hence the majority of the remaining drag reduction is sourced in these two regions.

\section{[Insert Figure 22.]}

Consistent with the behaviour of drag force, the introduction of MG\&RW leads to a reduction in the lift force acting on the SUV. Table 5 suggests that the strongest influence comes from the front split. This is reflected by the overall decrease in underbody pressure around and upstream of the front wheels seen in Figure 22a.

By analysing the flow fields around the SUV, explanations for the pressure variations can be identified. In Figures 23 and 24, streamlines are all seeded from the same locations and rendered with the corresponding plane's normal velocity 


\begin{tabular}{|l|c|c|}
\hline & MG\&RW - FG\&FW & WSO - MG\&RW \\
\hline$\Delta \mathrm{C}_{\mathrm{D}}$ & -0.015 & -0.005 \\
\hline Base $\Delta \mathrm{C}_{\mathrm{D}}$ & -0.003 & 0.000 \\
\hline Diffuser $\Delta \mathrm{C}_{\mathrm{D}}$ & -0.005 & +0.026 \\
\hline$\Delta \mathrm{C}_{\mathrm{L}}$ & -0.050 & -0.003 \\
\hline$\Delta \mathrm{C}_{\mathrm{L}}$ Front & -0.045 & -0.017 \\
\hline$\Delta \mathrm{C}_{\mathrm{L}}$ Rear & -0.005 & +0.014 \\
\hline
\end{tabular}

Table 5. The effect of moving ground and rotating wheels on force coefficients.

component. It should be noted that the streamlines are a representation of the time averaged flow.

Around the front left wheel, Figure 23, the rotation of the wheel acts against the direction of the flow entering the wheel arch and thus reduces the flow's resultant velocity. This is responsible for the increase of pressure previously identified in this region. Over the lower half of the wheel, the rotation has the opposite effect, as the rotation imparts additional momentum onto the flow. As a result the vortex which is formed around the exposed side of the wheel is strengthened and lowered towards the ground. This effect is consistent with the general trends identified by Waschle ${ }^{8}$, however the spoked rim used in their study generated secondary flow structures as flow passed through gaps in the wheel. The influence of rotation around the lower half of the wheel leads to smaller front wheel wakes and permits a greater mass flow under the front of the model. This is responsible for the overall lower underbody pressure over the front half of the model and reduction in front lift.

Figure 24 shows the flow behaviour around the rear left wheel and the influence of rotation on the flow entering the wake. The rotation changes the direction of the flow within the wheel arch, forcing the majority of flow inwards and through the gap between the wheel and SUV body. When this flow exits the wheel arch on the inboard side, an upwash of flow due to wheel rotation adds momentum to the flow over the lower portion of the wheel. This effect increases the mass flow entering the wake and is responsible for the increase in pressure on the base surface seen in Figure 21a.

\section{[Insert Figure 23.]}

[Insert Figure 24.]

Collectively, these results show the large influence that wheels have on the entire generic SUV flow field. Importantly, this is not limited to the rear wheel wakes and their impact on the base wake, but also the flow around the front wheels and within their housings.
The influence of wheel standoff (WSO - MG\&RW) A final comparison between MG\&RW and the experimental practice of wheel standoff (WSO) is now made. The values of overall $\Delta C_{D}$ and $\Delta C_{L}$ shown in Table 5 suggest that there is minimal difference between the two cases, however this is not the case when surfaces pressure are analysed. In Figure $21 \mathrm{~b}$ where $\Delta C_{p}=C_{p}^{\mathrm{WSO}}-C_{p}^{\mathrm{MG} \& \mathrm{RW}}$, the introduction of a standoff is shown to produce a lower pressure on the upper half and higher pressure on the bottom half of the base surface. Coincidently, when an area weighted base pressure is calculated, these differences balance.

On the underbody and wheel surfaces, large pressure differences can be found, Figures $22 \mathrm{~b}$ and 25 . The introduction of a standoff between the wheels and the ground allows flow to pass underneath the wheels, shown in Figures 23 and 24, thus the stagnation point at the front lower face of each wheel is removed. As well as contributing to the drag reduction, this standoff reduces the pressure upstream of the front wheels and consequently front lift coefficient.

\section{[Insert Figure 25.]}

This comparison is a useful example of how force coefficient values can be misleading. An analysis based purely on these values would infer that the experimental practice of a small wheel standoff can provide a good approximation of the flow around the same model with moving ground and rotating wheels. However, the detailed analysis performed in this study has shown a fundamental difference in the flow field that fortuitously balances out in the global coefficient values.

\section{Conclusions}

This study presents important comparison data for different CFD methodologies using commercial and open-source 
codes, assessing their validity and accuracy in predicting wake structure and base pressure, for a generic SUV geometry. It is clear from the flow visualisations presented that the steady state RANS governing equations, using available eddy viscosity turbulence models, are unsuitable for this application. The wheels and geometry of the rear end generates large time dependent fluctuations in the wake, which are not accurately modelled by the turbulence model. This result appears to be independent of code choice as does the computational expense, with broadly similar elapsed solver times between the commercial and open source codes.

A consequence of this result was the need to employ a transient solver. URANS showed limited improvements to the flow accuracy despite a significant increase in computational expense. A DES solver was found to offer considerable improvements in flow accuracy, albeit for a larger computational expense due to the reduction in time step size. This was seen through visual comparisons to the experimental PIV wake data. One of the most important results of this work was the high level of similarity between the solution obtained with the Lattice-Boltzmann Method, and the more widely used DES approaches. Flow structures within the wake showed little variation between the two approaches and as with the RANS results, differences were restricted to the running costs. Of the codes running DES, the open source code slightly outperformed the commercial code with lower run times, but was still unable to match the speed of LBM, running in approximately a fifth of the time of the commercial DES.

Errors in averaged drag coefficient when compared to the experiment were of similar values for the DES and LBM solvers, approximately $\pm 2 \%$, and of considerably smaller magnitude than the RANS and URANS solvers. This was expected due to the correlation between accurate wake prediction and drag accuracy. Variations in the lift coefficient existed across all configurations, but all agreed on the negative direction, in disagreement with the experiment. It is thought that this is due to the experimental procedure, which required a small clearance between the base of the wheels and the floor. It was postulated that failing to accurately capture these regions of high velocity flow in simulations would result in variations from the experiment on the underbody surface pressure values and hence lift coefficient. Experimental underbody surface pressure data is required to confirm this. Comparing the computational base pressures, the trend is maintained, with DES and LBM methodologies providing good agreement with each other but also with experiment.

With the findings of the code comparison study, an investigation into the influence of a moving ground and rotating wheels case (MG\&RW) was performed. An increase in base and diffuser pressure due to wheel rotation re-energising the wheel wakes was the primary result of the analysis and this contributed to approximately half of the total drag reduction. The remaining drag reduction was identified at the front wheels, where the rotation of the wheels reduced the resultant velocity of the flow in the front halves of the wheel arches and increasing the pressure. In addition, the moving ground permitted a greater mass flow under the vehicle reducing pressure, most notably around the front of the underbody. Consequently, this led to a reduction in front lift.

Finally, the effect on the experimental wheel standoff was investigated. Variations in drag and lift coefficients were minimal but misleading. Inspection of the surface pressures and flow field around the front and rear wheels highlighted this. Significant variations in underbody, base and diffuser pressures implied the similarities between force coefficient values were purely coincidental.

\section{Acknowledgements}

The authors would like thank HPC-Midlands grant EP/K000063/1 who facilitated the CFD simulations. This work was supported by Jaguar Land Rover and the UK EPSRC grant EP/K014102/1 as part of the jointly funded Programme for Simulation Innovation. All data created during this research are openly available from the Loughborough University data archive at https : / / dx . do i . org/10.17028/rd.1boro.3806823

\section{References}

1. Sterken L, Sebben S, Walker T, Lofdahl L. Experimental and Numerical Investigations of the Base Wake on an SUV. In: SAE Technical Paper. 2013-01-0464; 2013. p. 1-12.

2. Sterken L, Lofdahl L, Sebben S, Walker T. Effect of Rear-End Extensions on the Aerodynamic Forces of an SUV. In: SAE Technical Paper. 2014-01-0602; 2014. .

3. Sterken L, Sebben S, Lofdahl L, Walker T, Wolken T. Wake and Unsteady Surface-Pressure Measurements on an SUV with Rear-End Extensions. In: SAE Technical Paper. 2015-01-1545; 2015. p. 1-12. 
4. Wood A, Passmore M, Forbes D, Wood D, Gaylard A. Base Pressure and Flow-Field Measurements on a Generic SUV Model. SAE Int J Passen Cars - Mech Syst. 2015 Apr;8(1):1-9.

5. Nebenfuhr B. OpenFOAM: A tool for predicting automotive relevant flow fields. Chalmers University of Technology. Goteborg, Sweden; 2010.

6. Ashton N, Revell A. Comparison of RANS and DES Methods for the DrivAer Automotive Body. In: SAE Technical Paper. 2015-01-1538; 2015. p. 1-10.

7. Ashton N, West A, Lardeau S, Revell A. Assessment of RANS and DES methods for realistic automotive models . Computers and Fluids. 2016 Apr;128(C):1-15.

8. Waschle A. The Influence of Rotating Wheels on Vehicle Aerodynamics - Numerical and Experimental Investigations. In: SAE Technical Paper. 2007-01-0107; 2007. p. 1-17.

9. Duncan B, Kandasamy S, Sbeih K, Lounsberry TH, Gleason ME. Further CFD Studies for Detailed Tires using Aerodynamics Simulation with Rolling Road Conditions. In: SAE Technical Paper. 2010-01-0756; 2010. p. 1-19.

10. Kandasamy S, Duncan B, Gau H, Maroy F, Belanger A, Gruen $\mathrm{N}$, et al. Aerodynamic Performance Assessment of BMW Validation Models using Computational Fluid Dynamics. In: SAE Technical Paper. 2012-01-0297; 2012. p. 1-21.

11. Gleason ME, Duncan B, Walter J, Guzman A, Cho YC. Comparison of Computational Simulation of Automotive Spinning Wheel Flow Field with Full Width Moving Belt Wind Tunnel Results. SAE Int J Passen Cars - Mech Syst. 2015 Apr;8(1):1-19.

12. Le Good G, Garry K. On the Use of Reference Models in Automotive Aerodynamics. In: SAE Technical Paper. 200401-1308; 2004. p. 1-28.

13. Johl G, Passmore M, Render P. Design Methodology and Performance of an Indraft Wind Tunnel. The Aeronautical Journal. 2004 Sep;p. 465-473.

14. Kotapati R, Keating A, Kandasamy S, Duncan B, Shock $\mathrm{R}$, Chen H. The Lattice-Boltzmann-VLES Method for Automotive Fluid Dynamics Simulation, a Review. In: SAE Technical Paper. 2009-01-057; 2009. p. 1-13.

15. Landstrom C, Sebben S, Lofdahl L. Effects of Wheel Orientation on Predicted Flow Field and forces when modelling rotating wheels using CFD. In: 8th MIRA International Vehicle Aerodynamics Conference; 2010. p. 1-9.

16. Elofsson P, Bannister M. Drag Reduction Mechanisms Due to Moving Ground and Wheel Rotation in Passenger Cars . In: SAE Technical Paper. 2002-01-0531; 2002. p. 1-16.

17. Landstrom C, Lofdahl L. Detailed Flow Studies in Close Proximity of Rotating Wheels on a Passenger Car. SAE Int J Passen Cars - Mech Syst. 2009 Apr;2(1):861-874.
18. Koitrand S, Lofdahl L, Rehnberg S, Gaylard A. A Computational Investigation of Ground Simulation for a Saloon Car. SAE International Journal of Commercial Vehicles. 2014 Apr;7(1):111-123. 


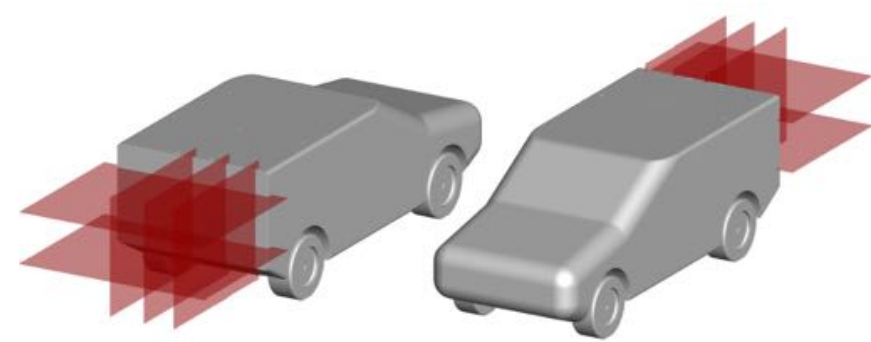

Figure 1. Generic SUV Model and Locations of PIV Planes.

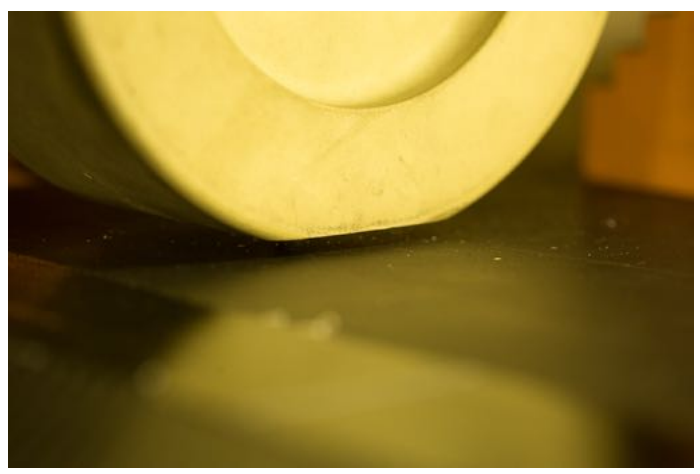

Figure 2. $4 \mathrm{~mm}$ Clearance between wheel and floor in the LUMS wind tunnel.

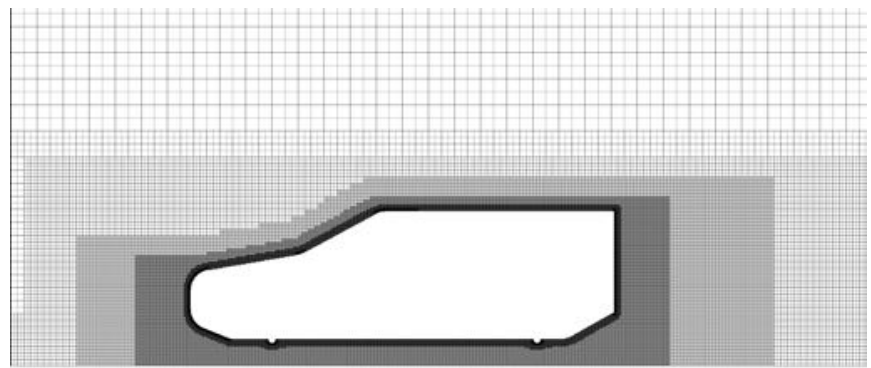

(a) Lattice

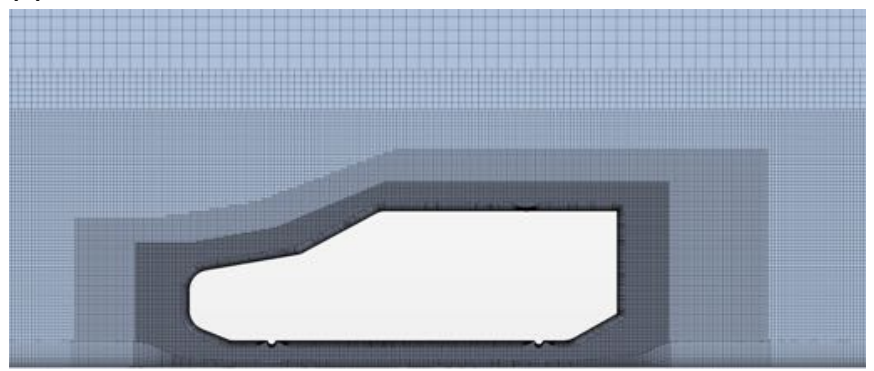

(b) Finite Volume Code Mesh

Figure 4. Areas of refinement for lattice and finite volume code mesh.

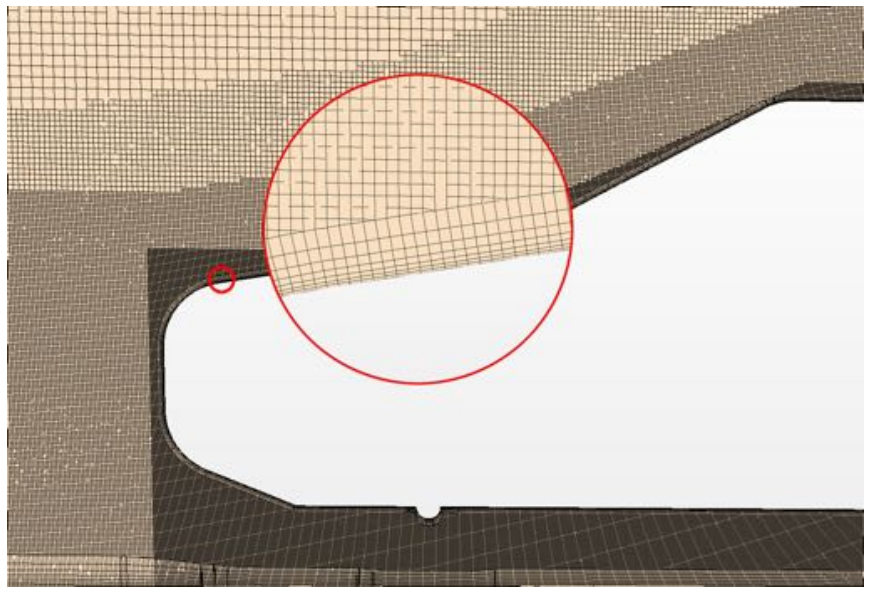

Figure 5. Finite volume code prism layers.

Figure 3. CFD Domain. 


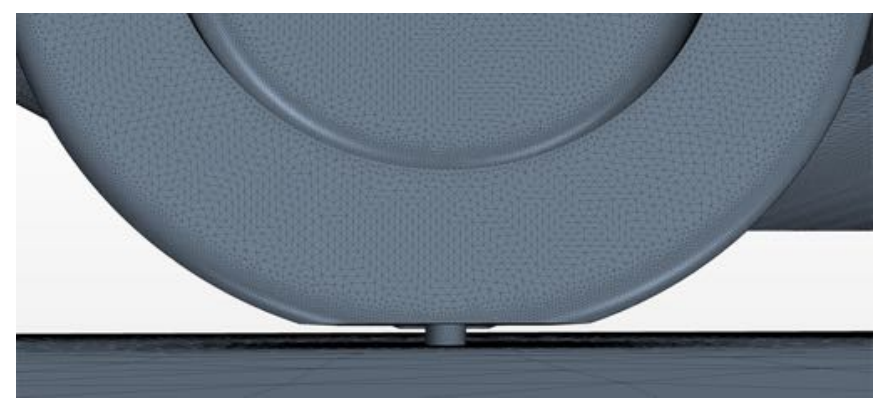

(a) With standoff

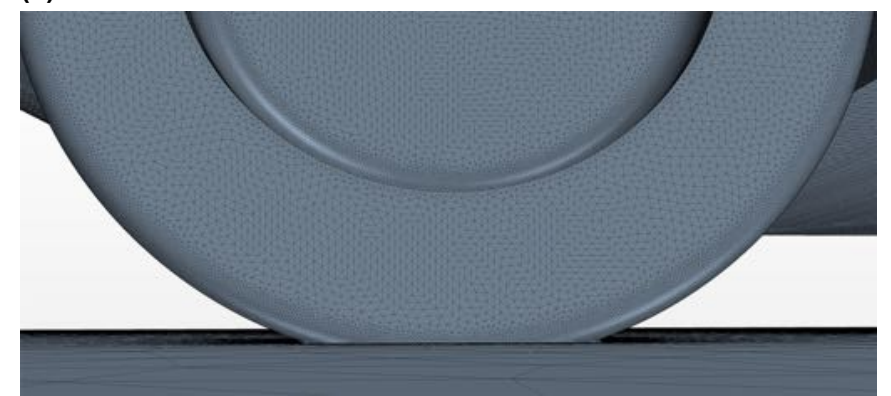

(b) With ground contact

Figure 6. Differences in wheel-ground contact

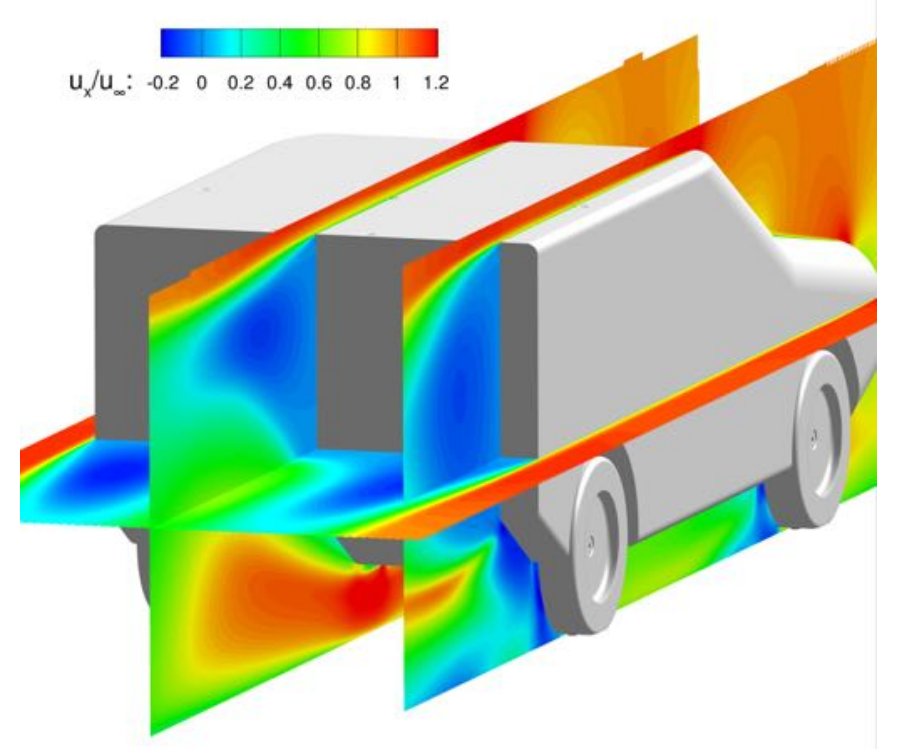

Figure 7. SUV flow field taken from commercial RANS

simulation (Vertical PIV Planes $Y=0, Y=0.17$ and Horizontal $Z=0.187$ ). 


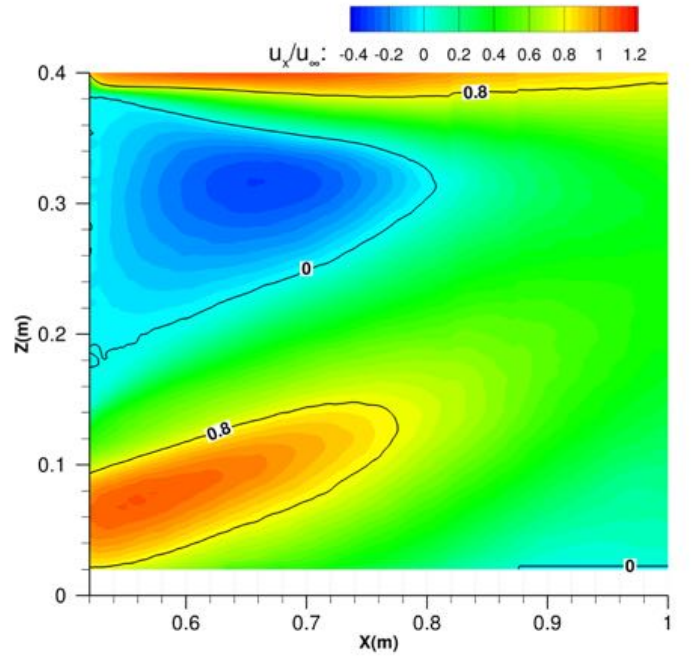

(a) Experiment, $Y=0$

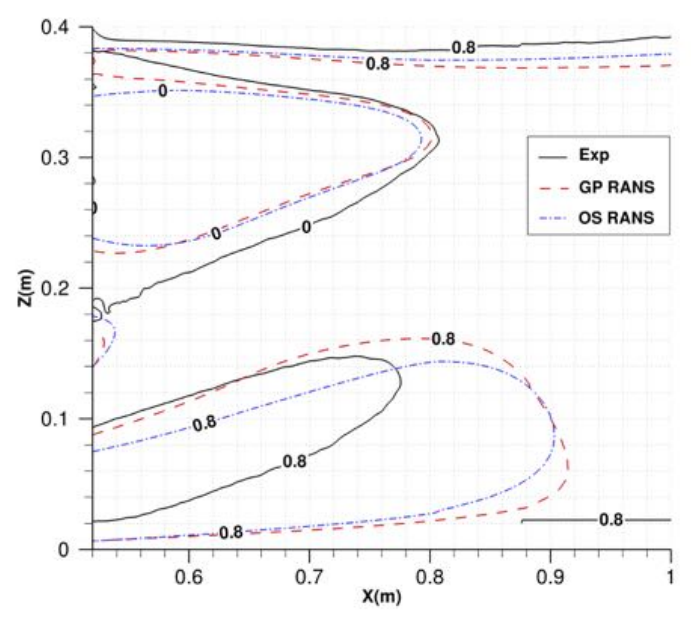

(b) RANS Contour Comparison, $Y=0$

Figure 8. Experiment and RANS comparison, $Y=0$ centreline plane, normalized $u$ velocity.

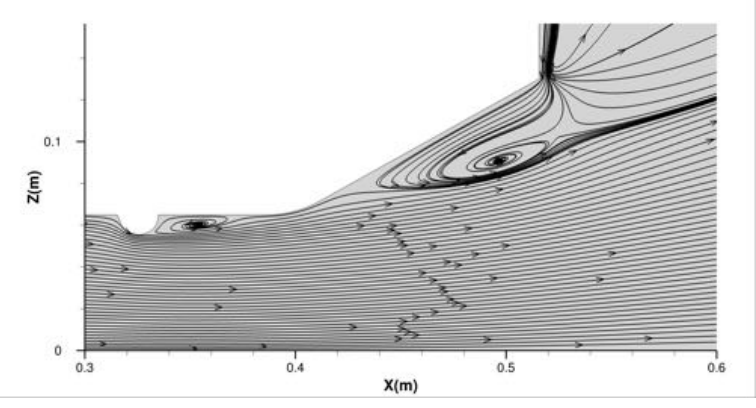

(a) RANS (Commercial Code)

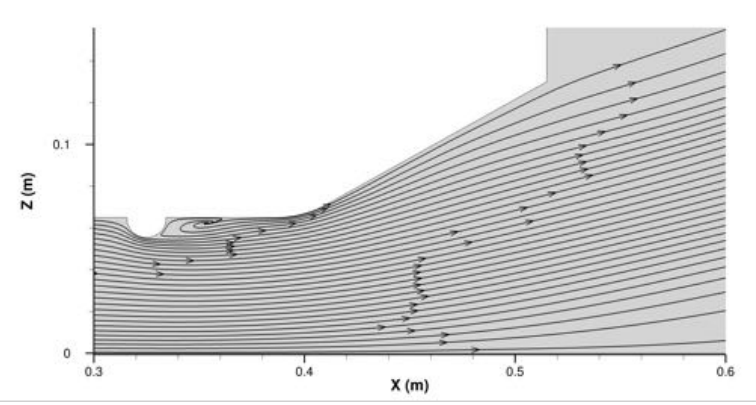

(b) DES (Commercial Code)

Figure 9. Flow streamlines along the centreline $(Y=0)$ and over the diffuser surface.

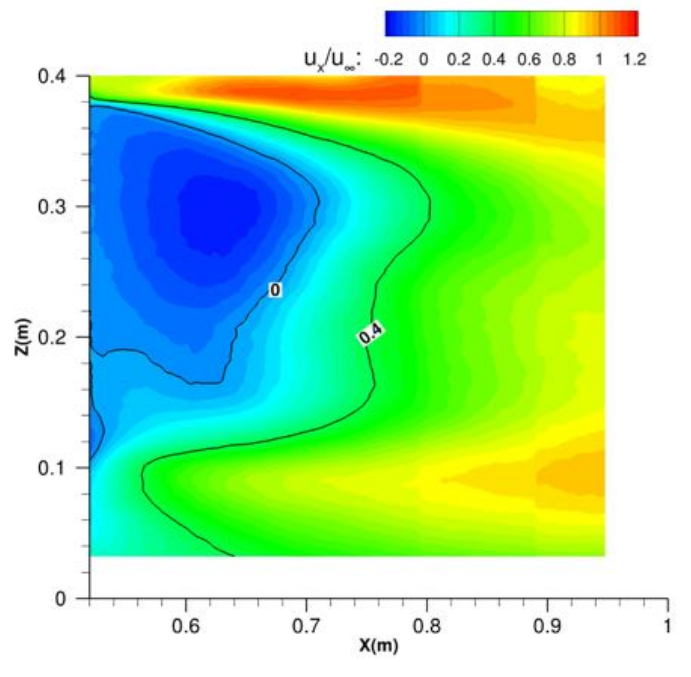

(a) Experiment, $Y=0.17$

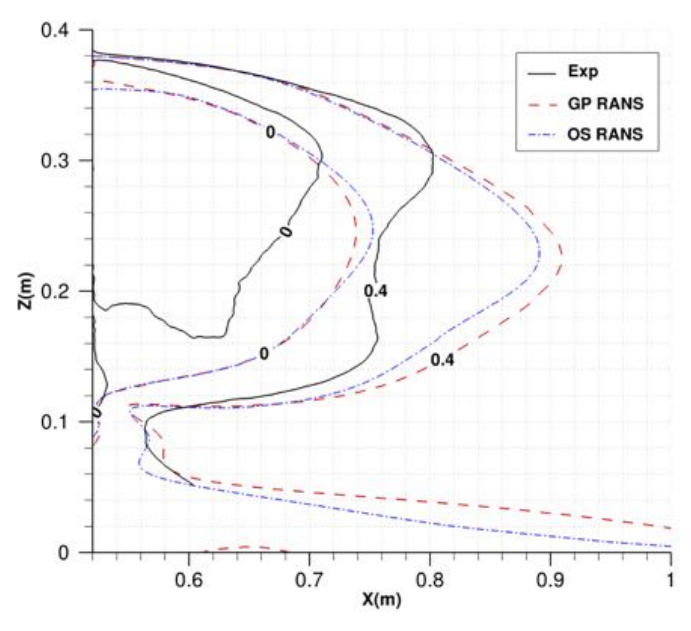

(b) RANS Contour Comparison, $Y=0.17$

Figure 10. Experiment and RANS comparison, $Y=0.17$ plane behind wheels, normalized $u$ velocity. 


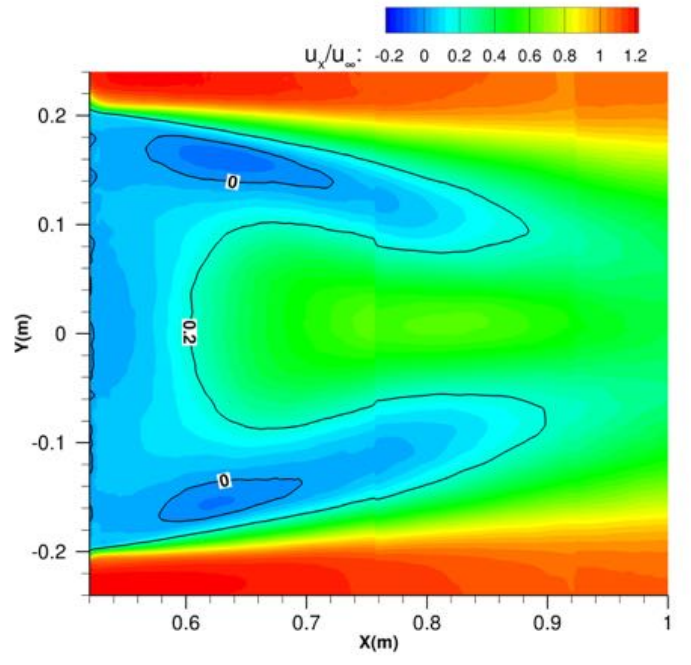

(a) Experiment, $Z=0.187$

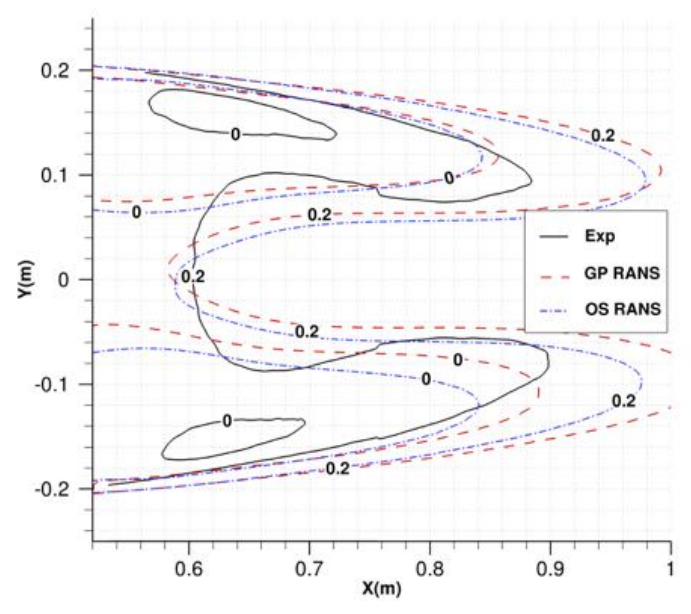

(b) RANS Contour Comparison, $Z=0.187$

Figure 11. Experiment and RANS comparison, $Z=0.187$ horizontal plane above diffuser, normalized $u$ velocity. 


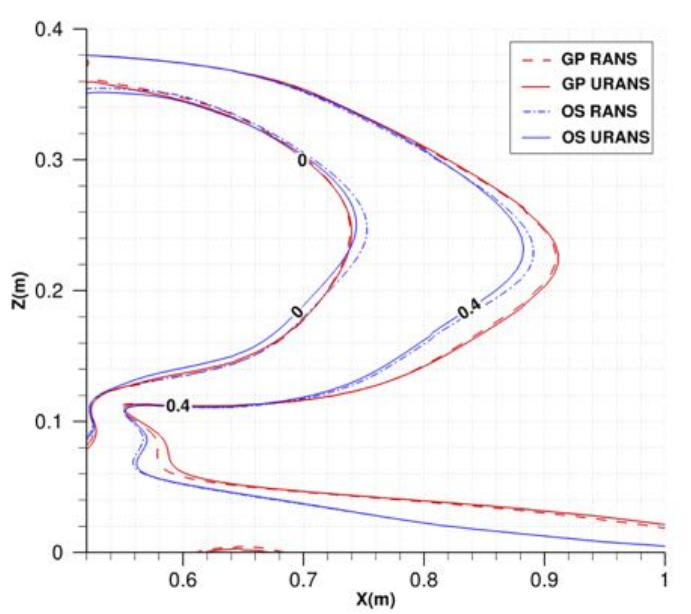

(a) RANS and URANS Contour Comparison, $Y=0.17$

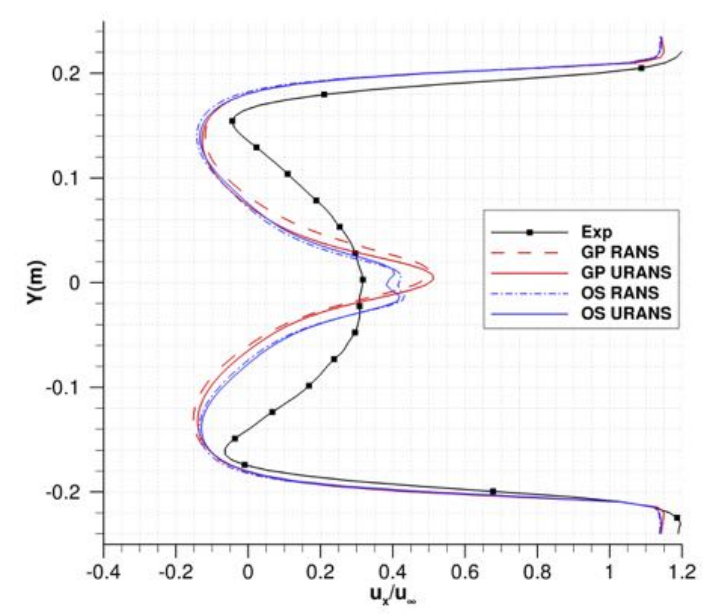

(b) Horizontal Line Probe of normalized $u$ velocity at $X=0.63, Z=0.187$

Figure 12. RANS and URANS comparison, $Y=0.17$ plane behind wheels, normalized $u$ velocity.

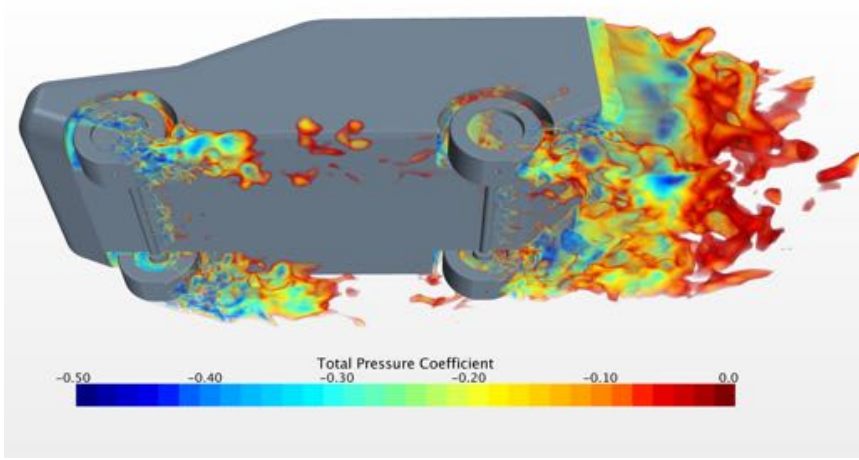

Figure 13. Instantaneous flow field around SUV, commercial, DES Solver. Volume threshold of $-0.5 \leq c_{p_{\text {total }}} \leq 0$.

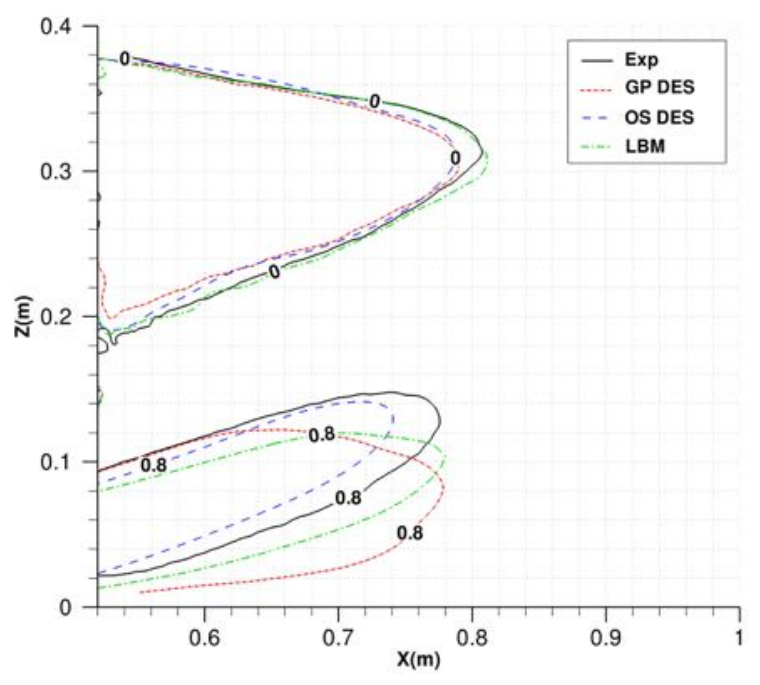

Figure 14. Experiment, DES and LBM Contour Comparison, $Y=0$ centreline plane, normalized $u$ velocity.

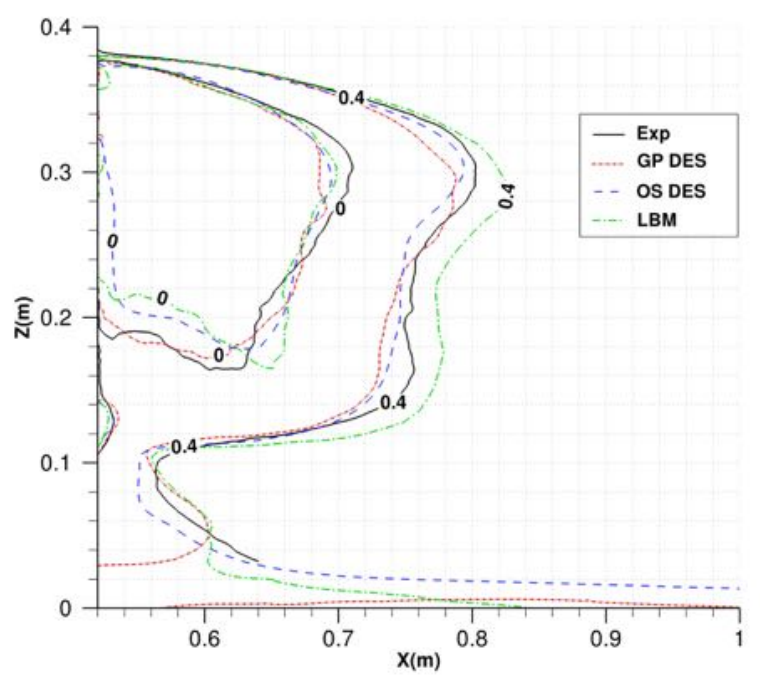

Figure 15. Experiment, DES and LBM Contour Comparison, $Y=0.17$ plane behind wheels, normalized $u$ velocity. 


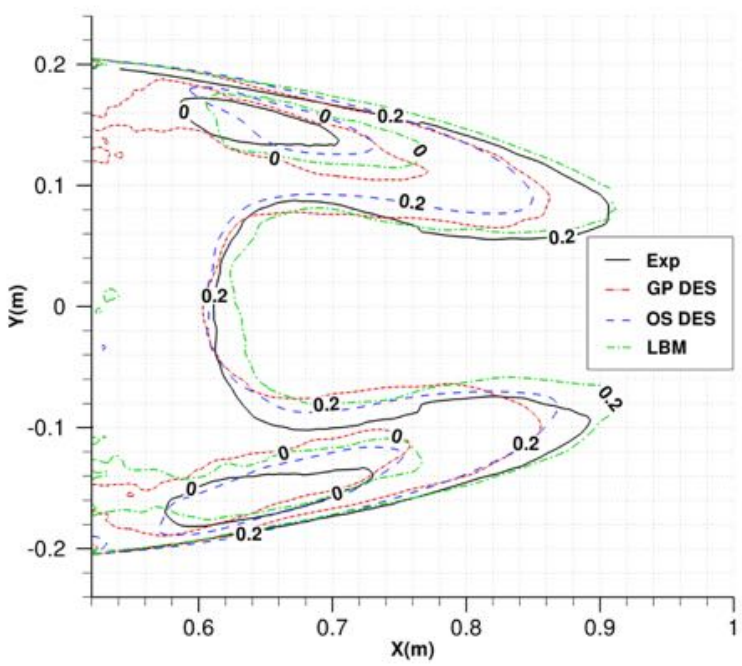

Figure 16. Experiment, DES and LBM Contour Comparison, $Z=0.187$ horizontal plane above diffuser, normalized $u$ velocity.

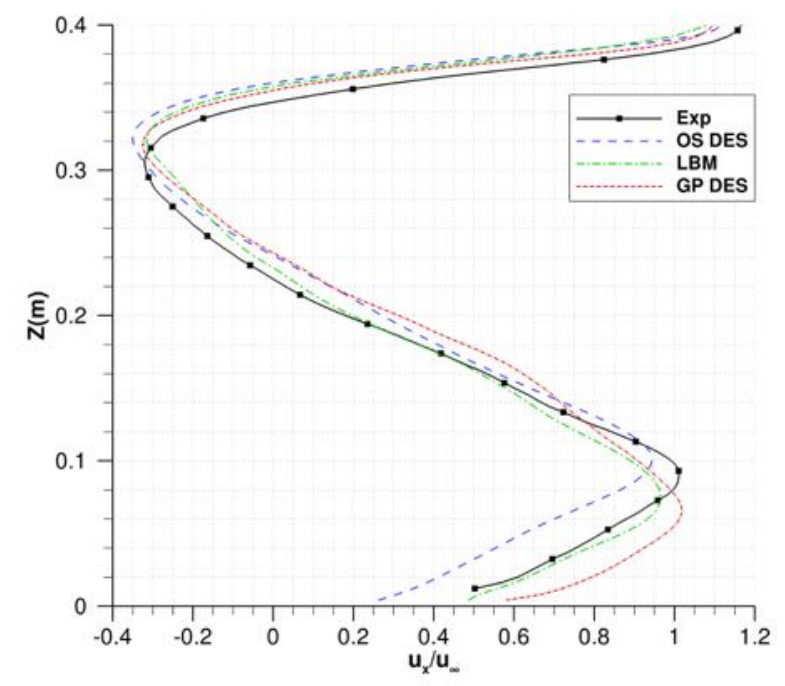

Figure 17. Vertical Line Probe of normalized $u$ velocity at $X=0.66, Y=0$

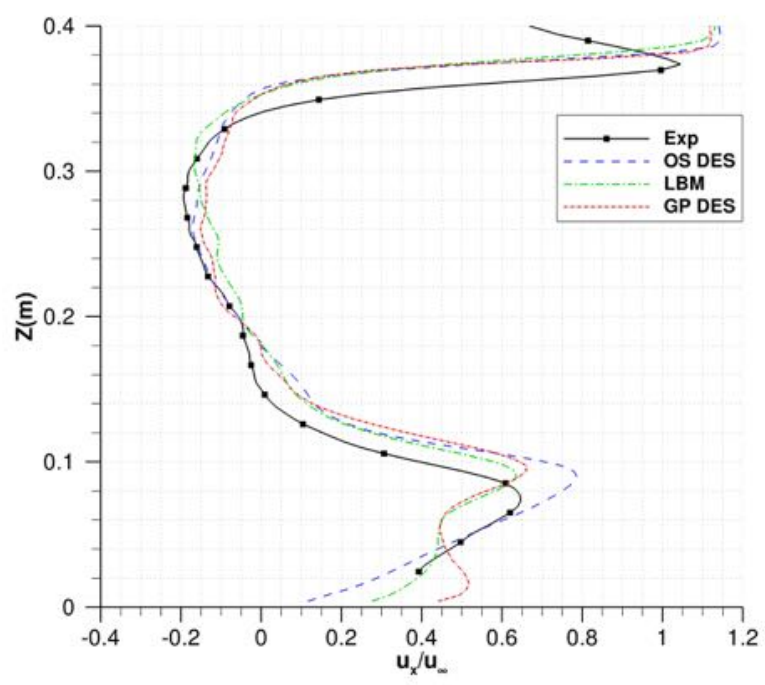

Figure 18. Vertical Line Probe of normalized $u$ velocity at $X=0.62, Y=0.17$

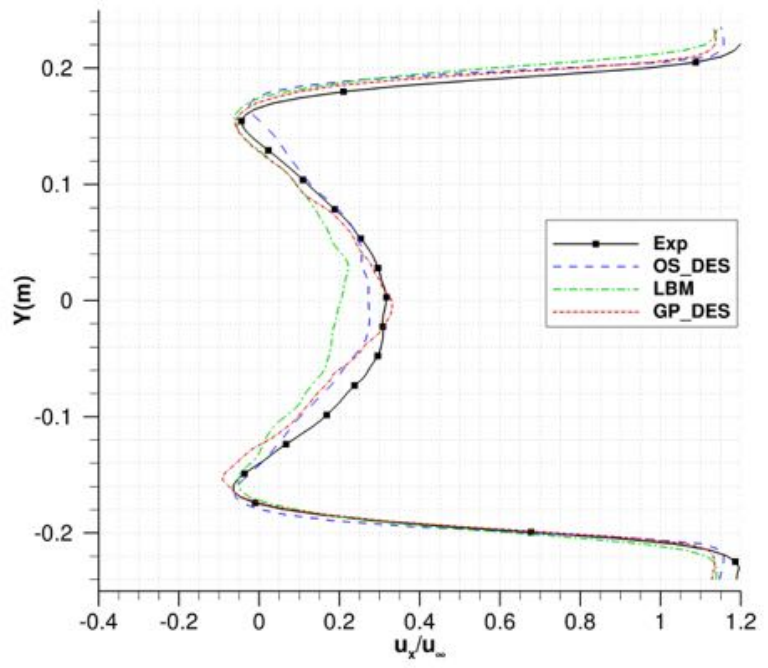

Figure 19. Horizontal Line Probe of normalized $u$ velocity at $X=0.63, Z=0.187$ 


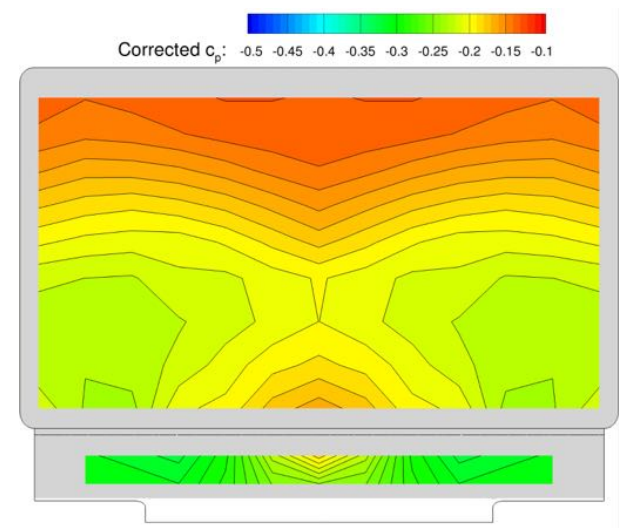

(a) Experiment

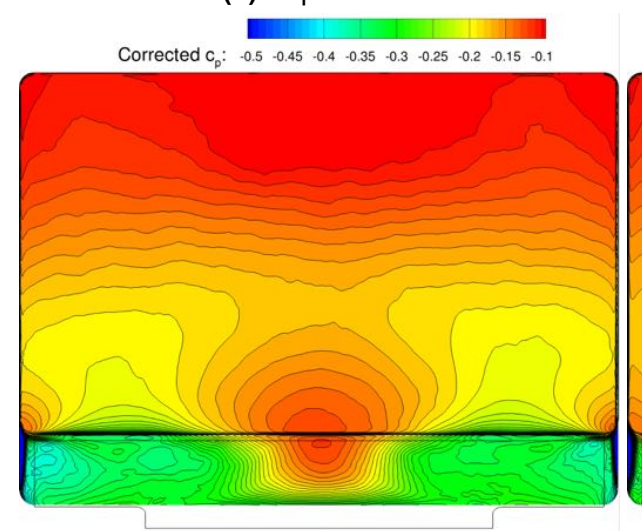

(c) Commercial Time Averaged DES

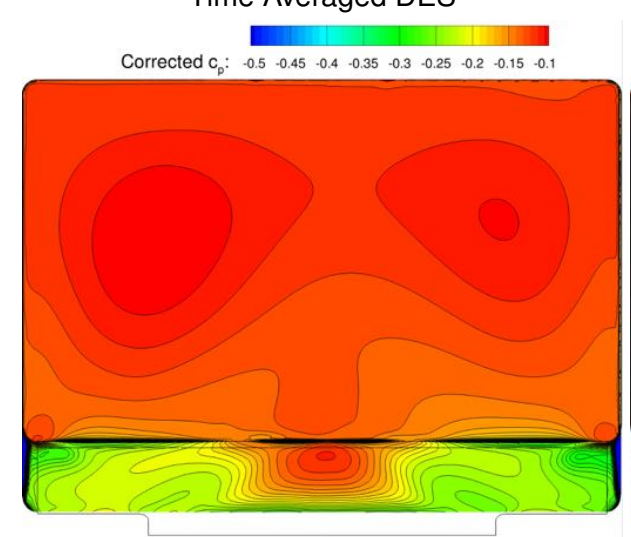

(e) Commercial RANS

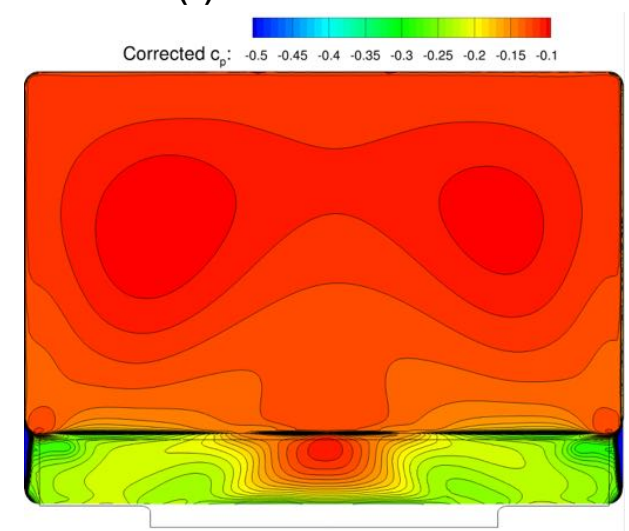

(g) Commercial Time Averaged URANS

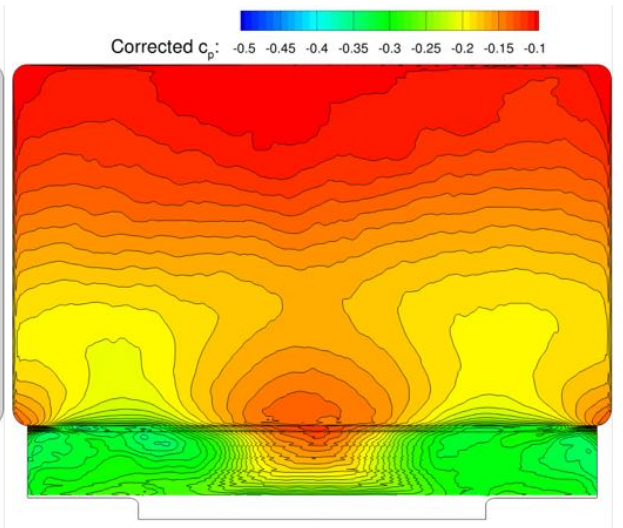

(b) Time Averaged LBM

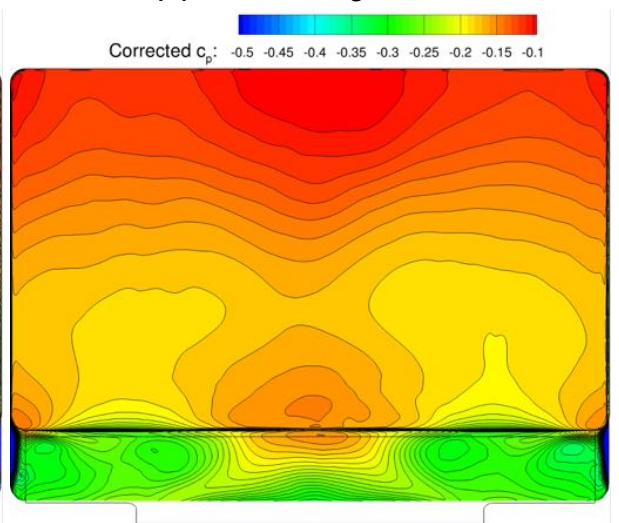

(d) Open Source

Time Averaged DES

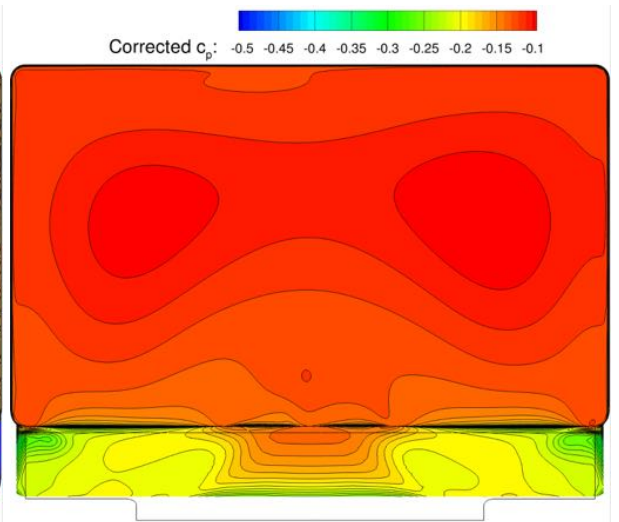

(f) Open Source RANS

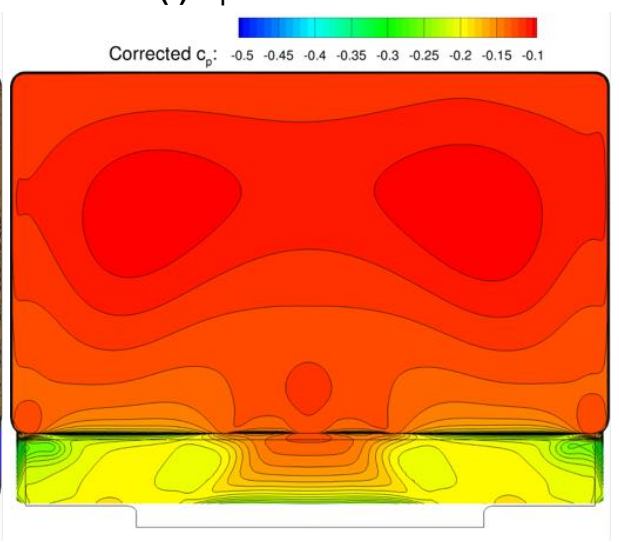

(h) Open Source Time Averaged URANS

Figure 20. Corrected surface base pressures. 
$\begin{array}{llllllllllll}\Delta \mathrm{C}_{\mathrm{p}}: & -0.02 & -0.016 & -0.012 & -0.008 & -0.004 & 0 & 0.004 & 0.008 & 0.012 & 0.016 & 0.02\end{array}$

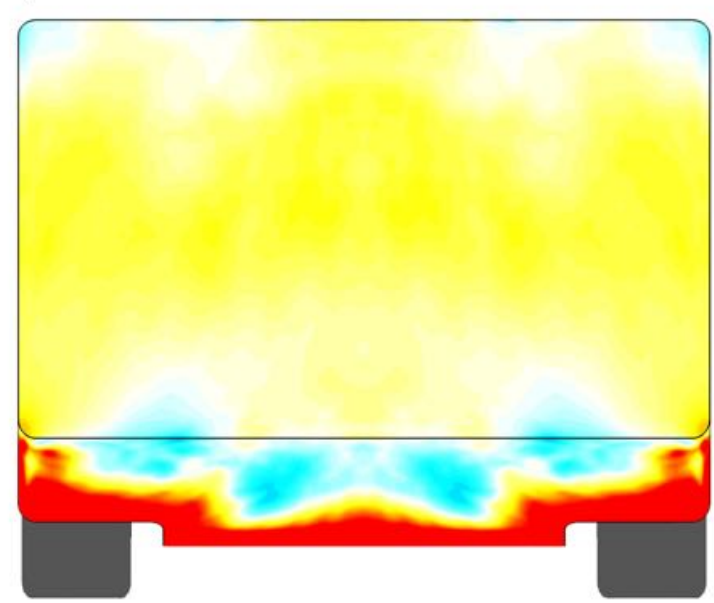

(a) MG\&RW - FG\&FW
$\Delta C_{\mathrm{p}}: \quad \begin{array}{cccccccccccc}0.02 & -0.016 & -0.012 & -0.008 & -0.004 & 0 & 0.004 & 0.008 & 0.012 & 0.016 & 0.02\end{array}$

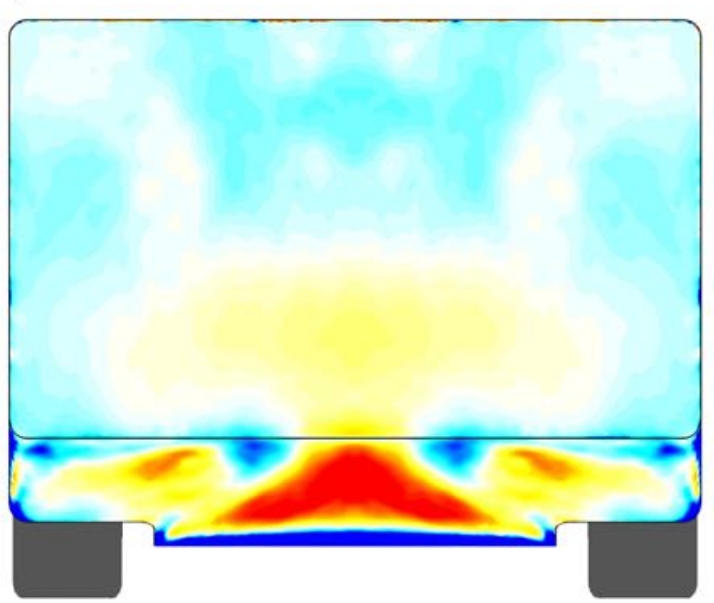

(b) WSO - MG\&RW

Figure 21. Differences in pressure coefficient, $\Delta C_{p}$, on base and diffuser surfaces
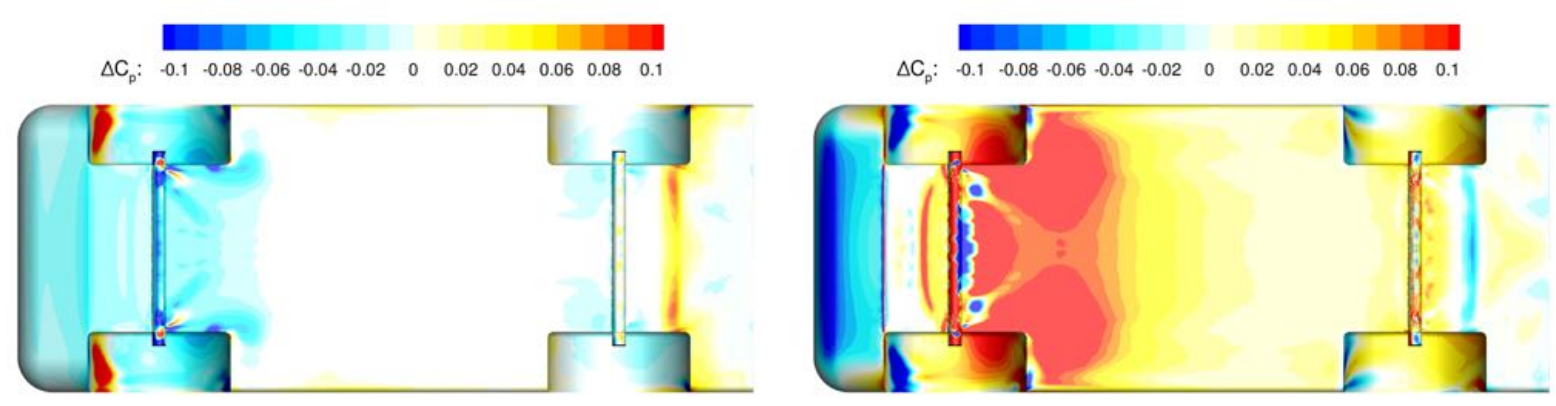

(a) MVG\&FW - FG\&FW

(b) WSO - MG\&RW

Figure 22. Differences in pressure coefficient, $\Delta C_{p}$, on the underbody surface and within wheel arches (wheels removed). 


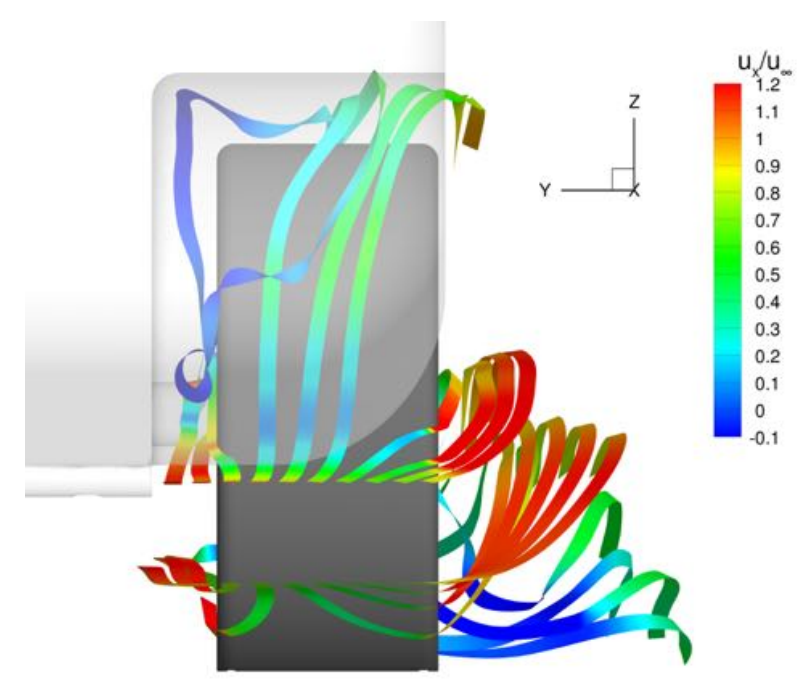

(a) FG\&FW

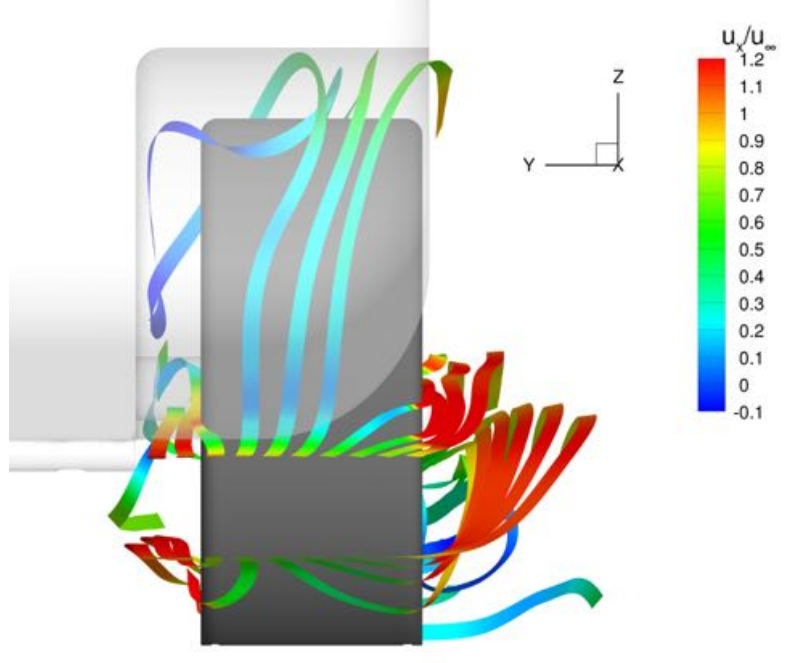

(c) MG\&RW

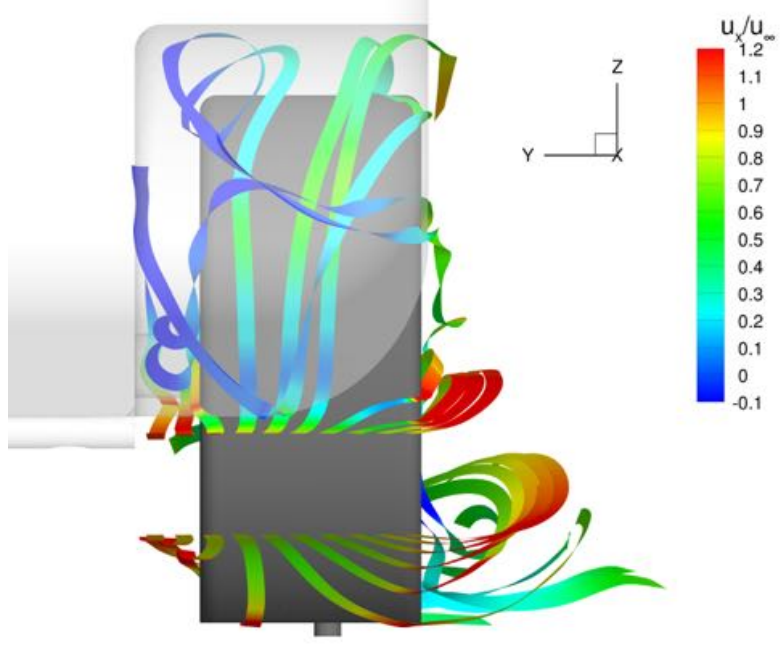

(e) WSO

Figure 23. Flow around front left wheel.

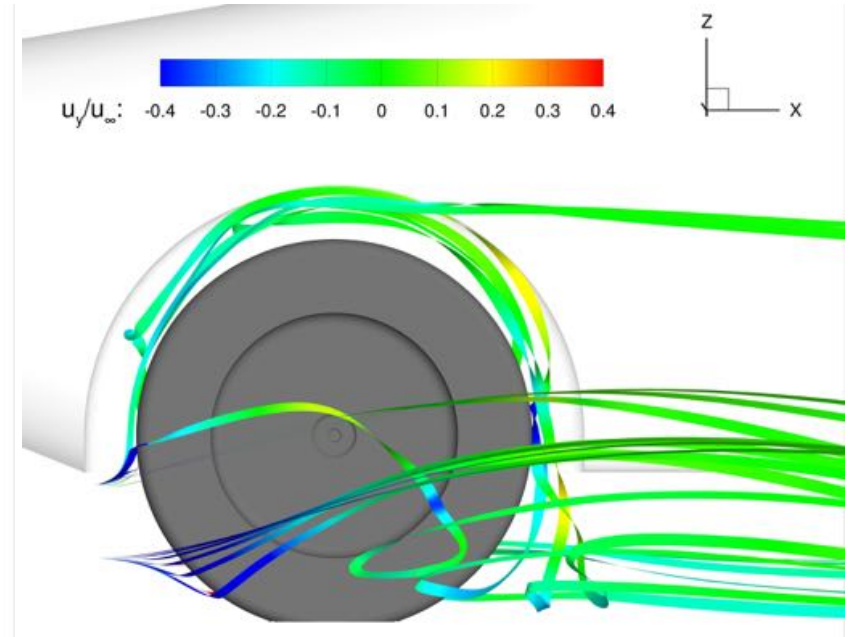

(b) FG\&FW

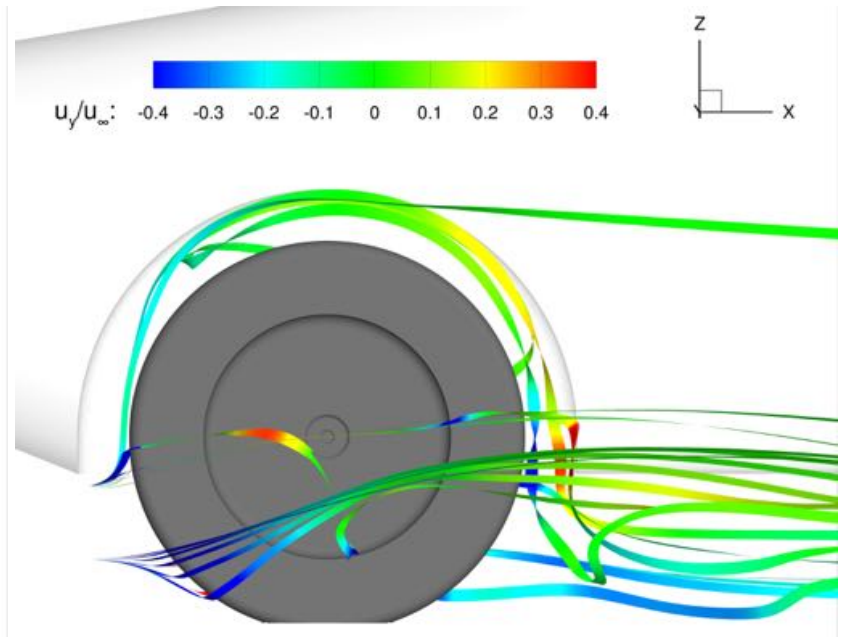

(d) MG\&RW

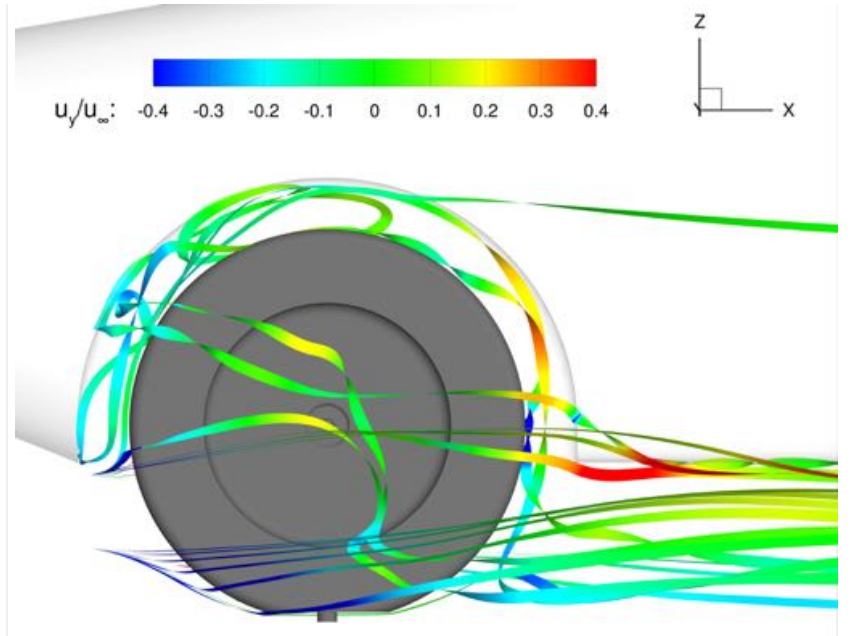

(f) WSO 


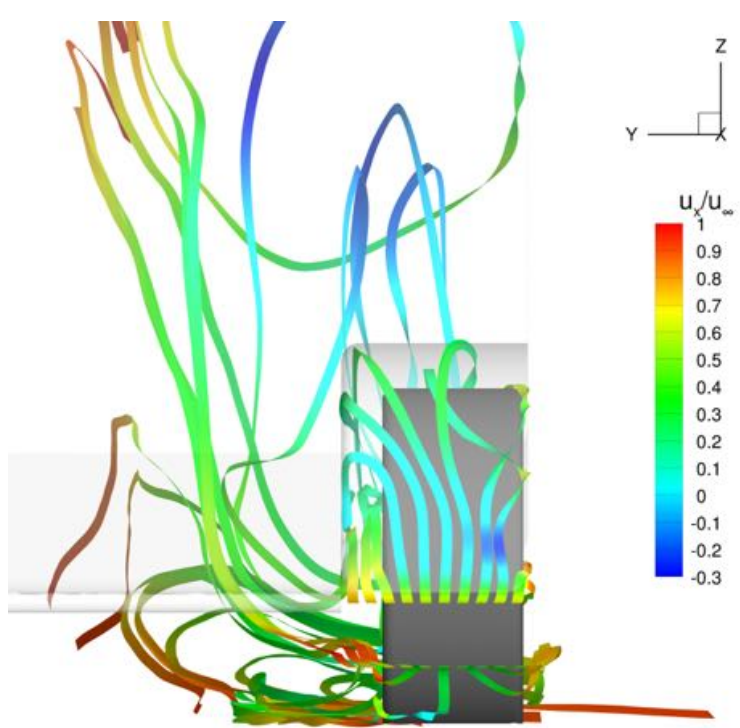

(a) FG\&FW

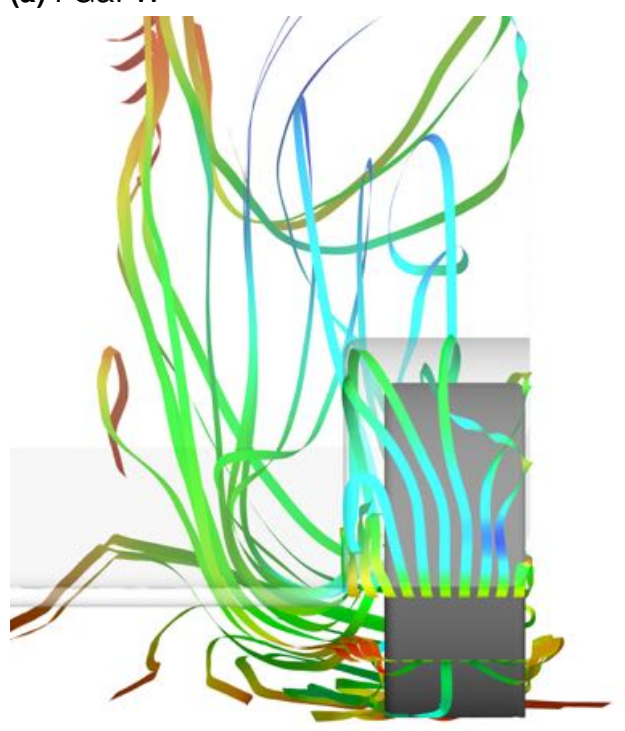

(c) MG\&RW

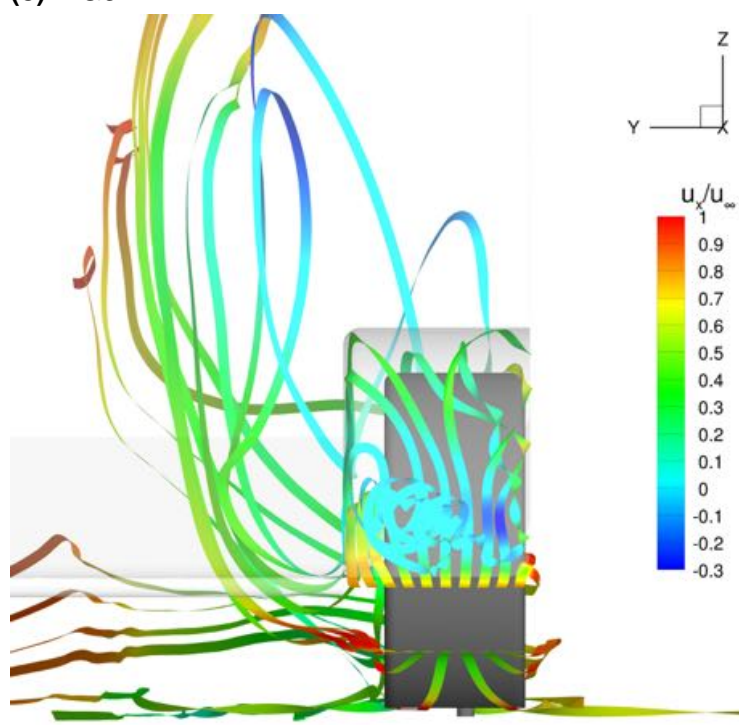

(e) WSO

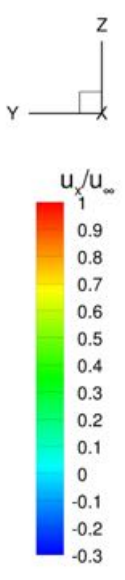

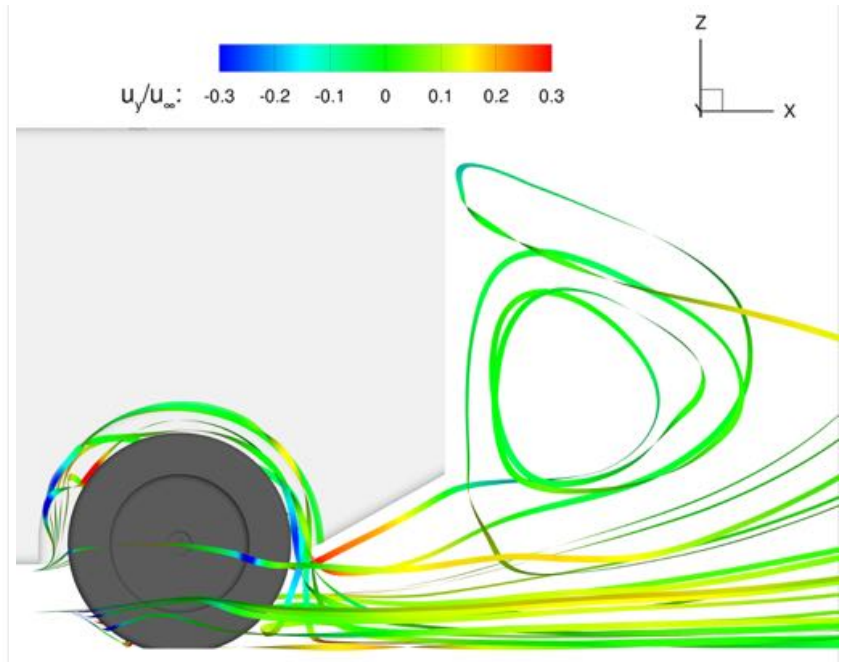

(b) FG\&FW

(d) MG\&RW

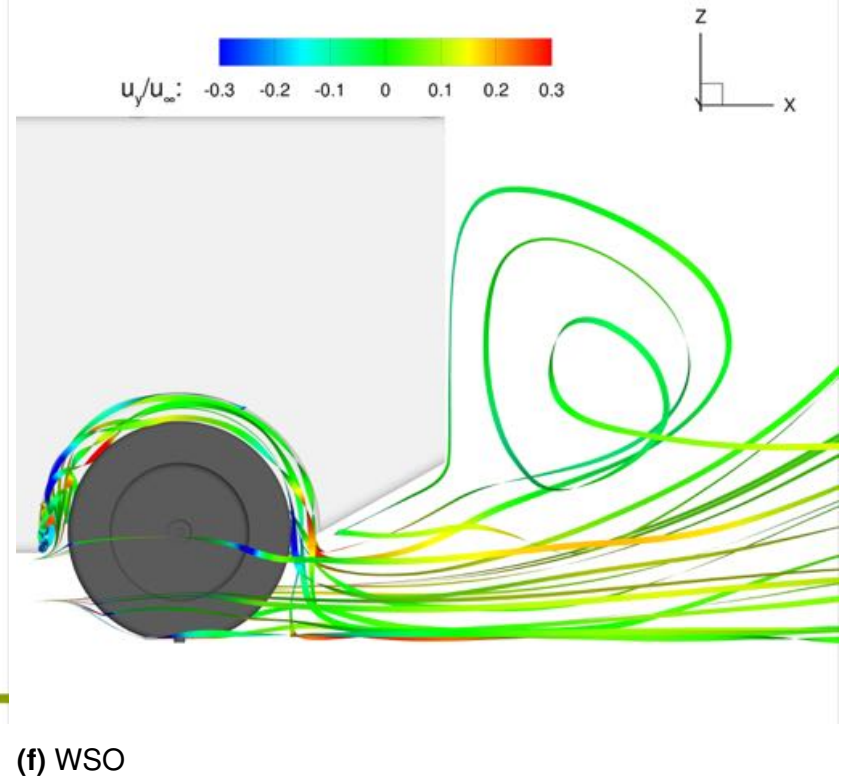

Figure 24. Flow around rear left wheel. 


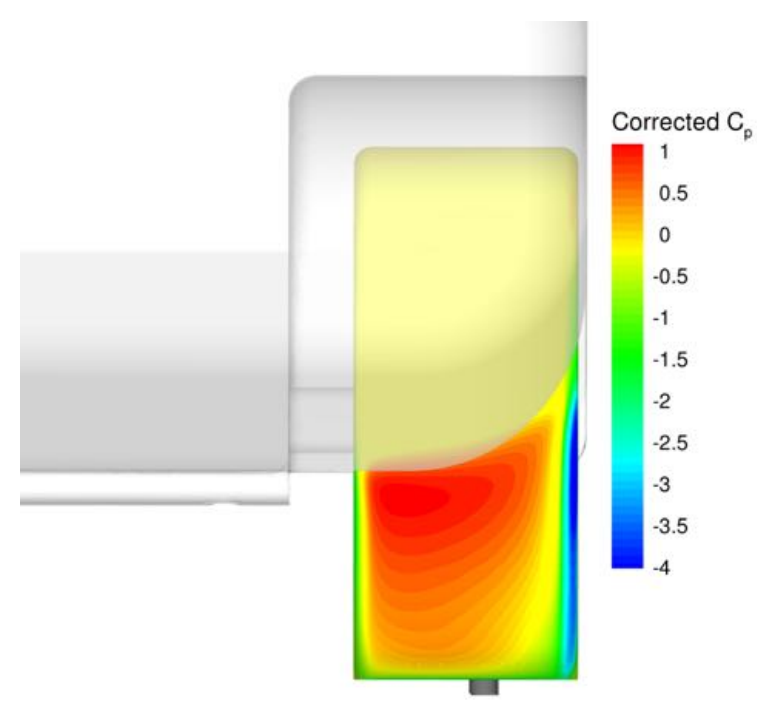

(a) WSO

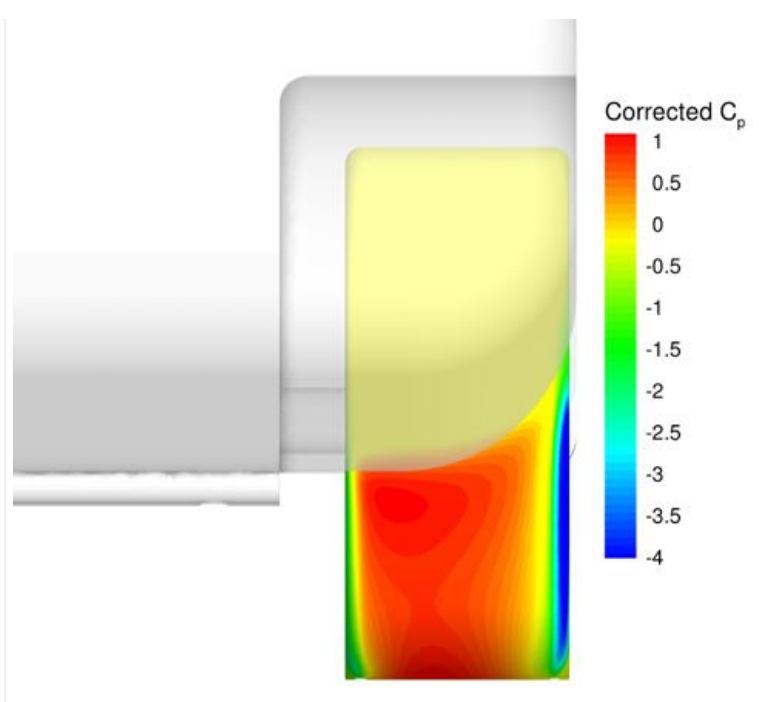

(b) MG\&RW

Figure 25. Pressure coefficient on forward facing, front left wheel surface. 